This item was submitted to Loughborough's Research Repository by the author.

Items in Figshare are protected by copyright, with all rights reserved, unless otherwise indicated.

\title{
Influence of tyre transience on anti-lock braking
}

PLEASE CITE THE PUBLISHED VERSION

PUBLISHER

Professional Engineering Publishing / @ IMECHE

VERSION

VoR (Version of Record)

LICENCE

CC BY-NC-ND 4.0

\section{REPOSITORY RECORD}

Jaiswal, Manish, George Mavros, Homer Rahnejat, and P.D. King. 2019. "Influence of Tyre Transience on Anti-lock Braking". figshare. https://hdl.handle.net/2134/6120. 
This item was submitted to Loughborough's Institutional Repository (https://dspace.lboro.ac.uk/) by the author and is made available under the following Creative Commons Licence conditions.

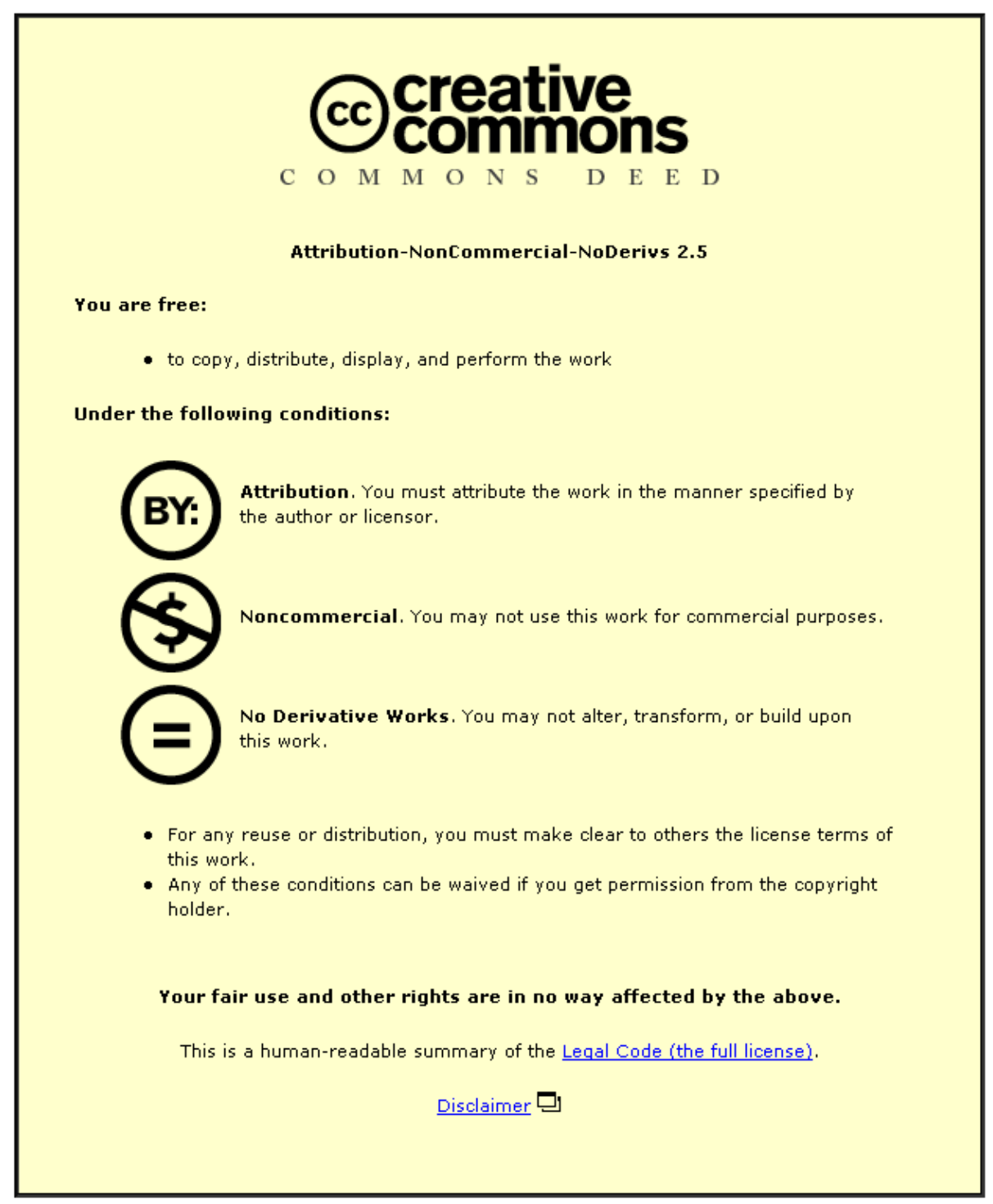

For the full text of this licence, please go to: http://creativecommons.org/licenses/by-nc-nd/2.5/ 


\title{
Influence of tyre transience on anti-lock braking
}

\author{
M Jaiswal $^{1}$, G Mavros ${ }^{2}$, H Rahnejat $^{1 *}$, and P D King ${ }^{1}$ \\ ${ }^{1}$ Wolfson School of Mechanical and Manufacturing Engineering, Loughborough University, Loughborough, \\ Leicestershire, UK \\ ${ }^{2}$ Department of Aeronautical and Automotive Engineering, Loughborough University, Loughborough, Leicestershire, UK
}

The manuscript was received on 31 May 2009 and was accepted after revision for publication on 4 August 2009.

DOI: 10.1243/14644193JMBD225

\begin{abstract}
Transient tyre characteristics can have significant influence in vehicle handling, particularly in anti-lock braking system (ABS), which involves wheel speed oscillations as a result of rapid changes in wheel brake pressure. Hitherto, ABS studies have been conducted mostly with straight-line motion. Relatively simple vehicle models have been used which cannot capture the interactions between non-linear handling dynamics and tyre behaviour. This article takes such interactions into account, using a non-linear 14-degrees-of-freedom vehicle model in combination with three different single-point contact tyre models with transient characteristics. They include a stretched-string-based model, a modified stretched-string model, and a contact mass model. The particularly demanding situation of combined cornering/ABS braking is investigated. It is shown that although all tyre models are of similar bandwidth (maximum frequency $\approx 15 \mathrm{~Hz}$ ), the simple string tyre model fails to cope with the non-linearities involved in combined braking/cornering and predicts greater braking distances than the two more enhanced tyre models.
\end{abstract}

Keywords: transient vehicle handling, anti-lock braking system braking, vehicle modelling, tyre transience

\section{INTRODUCTION}

Relaxation length is an important concept in studying tyre transient behaviour because of time delayed tyre response to various external inputs such as slip or camber. Tyre longitudinal and lateral flexibilities are the source of this time delayed response, which can be incorporated as first-order dynamic equations in vehicle-handling studies.

An early work by Owen and Bernard [1] concerned the study of build-up of lateral force on vehicle directional response, where the effect of tyre lateral flexibility was modelled as a first-order lag response to the changes in slip angle, using the relaxation length concept. Although their approach was quite straightforward, the model was not applicable for low speed because the vehicle speed appeared in the denominator of the equation, causing numerical instability.

\footnotetext{
*Corresponding author: Wolfson School of Mechanical and Manufacturing Engineering, Loughborough University, Loughborough, Leicestershire LE11 3TU, UK.

email:h.rahnejat@lboro.ac.uk
}

Ellis [2] also developed a lateral elastic tyre model for tyre transient response, where the time varying lateral deflection, in relation to the wheel rim, was modelled using a first-order differential equation. A relaxation time constant was used in the tyre lateral deflection, expressed as exponential time lag. Thus, the lateral tyre force, which is a product of lateral deflection and cornering stiffness, builds up to a steady-state value through an exponential function. Loeb et al. [3] attempted to quantify the relaxation length of a fully rolling steered tyre. They performed an analytical prediction of tyre transient response, using a single-point tyre contact model, employing a first-order differential equation, similar to the one used by Ellis [2]. Heydinger et al. [4] also used a firstorder dynamic equation, where the tyre side force was used as a variable instead of tread deflection, yielding a lagged side force response. Bernard and Clover [5] extended this approach to both longitudinal and lateral dynamics, using both longitudinal and lateral slip angles as time lagged variables for the respective first-order differential equations. By choosing slip as a variable, the authors addressed the numerical problem occurring at low vehicle speeds. 
Kuiper and Van Oosten [6] extended this approach to semi-empirical tyre models, by applying a stretchedstring model (based on the work of Pacejka [7]) to represent tyre transient behaviour, where the tyre belt is modelled as a stretched string, attached to the wheel rim by longitudinal and lateral springs. The tyre force and moments were then calculated using the standard magic formula coefficients with inclusion of transient slip as an input to the model.

In the past, several authors [8-11] have applied transient tyre models for anti-lock braking system (ABS) studies, where they have mainly used either quarter vehicle models or preliminary runs for straight-line braking. In another study, van Zanten et al. [12, 13] have conducted detailed experimental investigation, where they studied transients in longitudinal and lateral tyre forces in relation to an ABS system, using a vehicle fitted with a rotating wheel dynamometer. They further extended their study to develop a transient tyre model, which was simulated in a modelled tyre test stand to validate their experimental findings. Therefore, most of the aforementioned studies have tried to establish the effectiveness of particular transient tyre models to study responses in relation to the high-frequency input such as road undulations and brake torque variations. However, much scope still remains for studying tyre interactions with antilock braking, particularly during combined cornering and braking manoeuvres, where due to interaction of handling and braking, the influence of tyre transience may significantly deviate from those for straight-line braking. Conducting such a study in a representative full vehicle-tyre-ABS integrated set-up may not only aid in better prediction of the tyre behaviour in more demanding situations, but also aid establishment of transient tyre behaviour in vehicle braking and stability control studies.

\section{THE VEHICLE MODEL}

The vehicle model used in this study is an extension of that reported in Jaiswal et al. [14]. This is an intermediate model, based on Newton-Euler formulation. The vehicle model has already been validated against transient handling manoeuvres on a test track as well as a more complex multi-body model created in ADAMS [14].

The primary dynamics of the vehicle model is that of the six motions of the sprung mass in space. The complete vehicle model including the sprung mass and four unsprung masses consisting of the wheels, tyres, and parts of the suspension is shown in Fig. 1. While the sprung mass is allowed six degrees-of-freedom (DOF), the unsprung masses move only in the vertical direction with wheel rotations. The equations of motion of the sprung mass are derived according to Ellis [2] with respect to the vehicle SAE frame of reference, also shown in Fig. 1. The vehicle is assumed to enjoy geometrical symmetry about the $X Z$-plane of the SAE frame. However, the general case is considered, in which the vehicle is not inertially symmetrical about the same plane (i.e. the various products of inertia do not diminish). The only restriction adopted is that the origin of the SAE frame lies at the same longitudinal position as the centre of gravity (cg) of the vehicle. This requirement has no mathematical significance and is considered only to achieve general comparability of the results with the implications of analytical results obtained by simple bicycle models, where the origin of the SAE frame is usually taken at the same position [15]. Thus, the equations of motion for the three translational and three rotational DOF are

$$
\begin{aligned}
\Sigma F_{x}= & m_{\mathrm{T}}(\dot{U}-V r+W q)-m_{\mathrm{s}}\left[x_{\mathrm{G}}\left(q^{2}+r^{2}\right)\right. \\
& \left.-y_{\mathrm{G}}(p q-\dot{r})-z_{\mathrm{G}}(p r+\dot{q})\right] \\
\Sigma F_{y}= & m_{\mathrm{T}}(\dot{V}-W p+U r)-m_{\mathrm{s}}\left[y_{\mathrm{G}}\left(r^{2}+p^{2}\right)\right. \\
& \left.-z_{\mathrm{G}}(q \cdot r-\dot{p})-x_{\mathrm{G}}(p \cdot q+\dot{r})\right] \\
\Sigma F_{z}= & m_{\mathrm{s}}(\dot{W}-U q+V p)-m_{\mathrm{S}}\left[z_{\mathrm{G}}\left(p^{2}+q^{2}\right)\right. \\
& \left.-x_{\mathrm{G}}(p r-\dot{q})-y_{\mathrm{G}}(q r+\dot{p})\right] \\
\Sigma M_{x}= & I_{x x} \dot{p}-\left(I_{y y}-I_{z z}\right) q r+I_{y z}\left(r^{2}-q^{2}\right) \\
& -I_{z x}(p q+\dot{r})+I_{x y}(p r-\dot{q})+m_{\mathrm{S}} y_{\mathrm{G}} \\
& \times(\dot{W}-U q+V p)-m_{\mathrm{S}} z_{\mathrm{G}}(\dot{V}-W p+U r) \\
\Sigma M_{y}= & I_{y y} \dot{q}-\left(I_{z z}-I_{x x}\right) p r+I_{x z}\left(p^{2}-r^{2}\right) \\
& -I_{x y}(q r+\dot{p})+I_{y z}(q p-\dot{r})+m_{\mathrm{S}} z_{\mathrm{G}} \\
& \times(\dot{U}-V r+W q)-m_{\mathrm{S}} x_{\mathrm{G}}(\dot{W}-U q+V p) \\
\Sigma M_{z}= & I_{z z} \dot{r}-\left(I_{x x}-I_{y y}\right) p q+I_{x y}\left(q^{2}-p^{2}\right) \\
& -I_{y z}(r p+\dot{q})+I_{z x}(r q-\dot{p})+m_{\mathrm{T}} x_{\mathrm{G}} \\
& \times(\dot{V}-W p+U r)-m_{\mathrm{T}} y_{\mathrm{G}}(\dot{U}-V r+W q) \\
& (\dot{W}-V r)
\end{aligned}
$$

In the above equations $U, V$, and $W$ denote the three translational velocities of the sprung mass along the $X$-, $Y$-, and $Z$-axes of the SAE frame, respectively, while $p, q, r$ are the rotational speeds (roll, pitch, and yaw) about the same axes. The left-hand-side terms in equations (1) to (6) denote the net forces in the $X$-, $Y$-, and $Z$-directions, or the net moments about the same axes. In terms of inertial properties, $m_{\mathrm{T}}$ denotes the total mass of the vehicle, including the mass of the unsprung components. It is interesting to note that the longitudinal and lateral force equations (1) and (2) employ the total mass $\left(m_{\mathrm{T}}\right)$ terms, as the longitudinal and lateral DOF are subject to the vehicle's total inertia. On the other hand, when dealing with the vertical force (equation (3)), it is more appropriate to use only the sprung mass term $\left(m_{\mathrm{S}}\right)$, as the vertical dynamics 


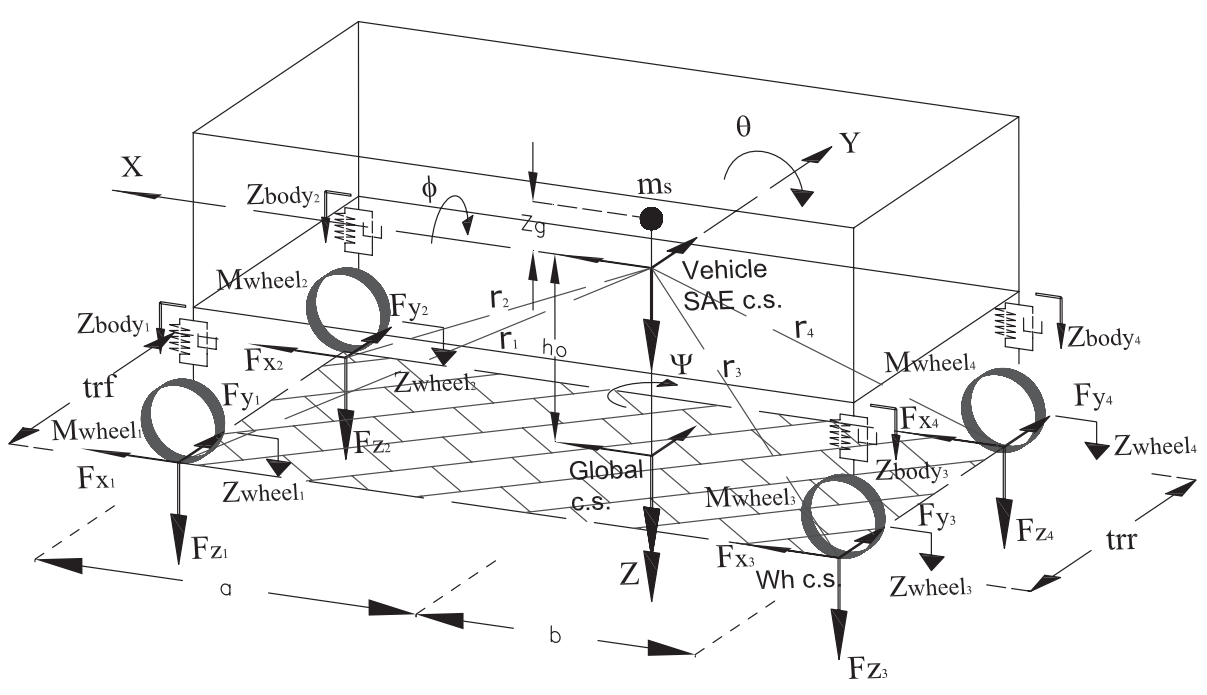

Fig. 1 The vehicle model, including the SAE frame of reference

of the unsprung mass is considered separately. Similarly, while writing equations for the net roll and pitch moments, only the sprung mass term $\left(m_{\mathrm{S}}\right)$ appears (refer to equations (4) and (5)), whereas in the case of yaw moment (equation (6)), the total vehicle mass $\left(m_{\mathrm{T}}\right)$ is considered.

An alternative and mathematically better approach would be to use the sprung mass $m_{\mathrm{S}}$ throughout equations (1) to (6) and provide all unsprung masses with additional lateral and longitudinal DOF. This would bring the model closer to its complex multi-body alternative, increasing unnecessarily the computational cost of simulation. It is important to emphasize that the treatment of mass presented herein is a simplification that aims to distribute the mass more appropriately between various DOF, without increasing their number and without introducing additional constraints. In the same spirit, parameters $x_{\mathrm{G}}, y_{\mathrm{G}}$, and $z_{\mathrm{G}}$ indicate the distance of the cg of the complete vehicle from the origin of the SAE frame of reference.

Finally, $I_{x x}$ and $I_{y y}$ indicate the sprung mass moments of inertia about the $X$ - and $Y$-axes, whereas $I_{z z}$ denotes the moment of inertia of the full vehicle about the $Z$-axis. The products of inertia $I_{x y}$, $I_{z x}$, and $I_{y z}$ are all calculated considering the sprung mass only. The forces on the left-hand side of equations (1) to (3) include those developed at the tyres' contact patches, the gravitational forces, suspension component forces, as well as the aerodynamic forces.

In terms of vertical dynamics, the four unsprung masses, which include wheels, tyres, and suspensions, are modelled as mass-spring-damper systems. The tyres are considered as linear spring-damper systems in the vertical direction which connect the unsprung masses to the road. At their top, the unsprung masses are connected to the vehicle body using springs and dampers representing non-linear wheel rates and damping functions. The total suspension force at each unsprung mass includes the effect of springs and dampers, anti-roll bar, and bump stops. In addition, the forces through the suspension links are indirectly modelled, using the principle of virtual work as described in Jaiswal et al. [14] and Azman et al. [16]. Non-linearities result from the geometry of suspension and are treated using the suspension-ratio concept [17] and also from the consideration of different bump/rebound damper settings.

\section{ABS BRAKING}

The ABS used here is based on a conventional peak seeking approach, where the wheel angular deceleration and slip thresholds are used to determine the brake control cycle, which is conceptually based on the published work by Bosch [18]. Figure 2 shows plot of the ABS control cycle used in this work. The ABS brake system is integrated with the intermediate vehicle model through MATLAB/Simulink environment and the $S$-function, which externally interfaces the ABS control algorithm (written in a $C$ code).

The ABS control algorithm used in this work relies more on the concept rather than the exact nature of the data, as the focus is to apply a representative ABS system for a tyre transient study, as opposed to using a detailed ABS system for design or performance-related studies. Additionally, instead of modelling the dynamics of the ABS hydraulic modulator, the algorithm takes into account the intrinsic characteristics of the modulator in a simplified representative manner. These characteristics include delays associated with the opening and closing of solenoid valves, applied brake pressure and release rates, applied delay while the solenoid is in the pressure holding phase, and also the characteristics of a pulse train, where the solenoid valve holds and builds up the pressure in a stepwise 


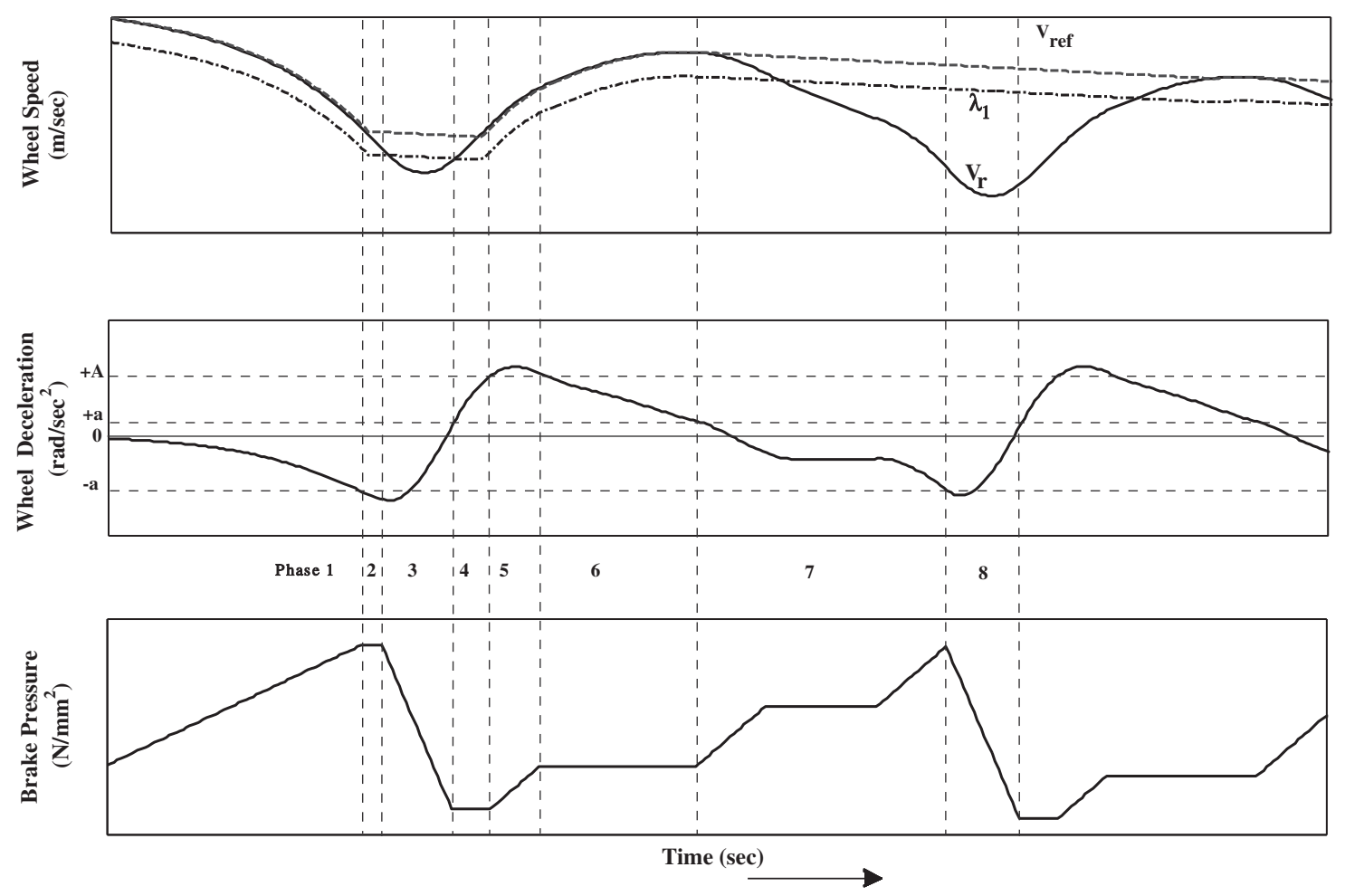

Fig. 2 ABS control cycle

manner. In the ABS control cycle adopted here, the wheel angular deceleration is used as a control variable rather than the wheel peripheral deceleration. This has no conceptual basis and is simply used as a convenience as the wheel rotational speed can be directly differentiated without multiplying it with the effective tyre rolling radius. However, the differentiating circuit in a control system often employs filters for signal processing. This means a cut-off frequency is needed, which can filter out the noise at a lower frequency, such that any faulty deceleration threshold can be avoided. Here, a low-pass filter is chosen with a cut-off frequency of $15 \mathrm{~Hz}$.

In the operational cycle, the brake pressure application is divided into eight phases, based on different brake pressure gradients. Phase 1 shows the start of the control cycle, where the output brake pressure is kept equal to the input pressure (from the master cylinder), until the wheel's peripheral deceleration goes beyond the defined threshold $(-a)$ at the end of this phase. In phase 2, the brake pressure is held constant as the solenoid valves move to the hold pressure position. The brake pressure is not reduced in this phase as the deceleration threshold $(-a)$ could have been exceeded with the coefficient of friction still in the stable range of the $\mu$-slip curve. This phase continues until the wheel's peripheral velocity $V_{\mathrm{r}}$ exceeds the slip threshold velocity $\lambda_{1}$. The slip threshold velocity is based on the reference velocity $V_{\text {Ref }}$, which is determined by a preset deceleration and hence reduces at the start of phase 2 . In phase 3 , the brake pressure is reduced as the solenoid valve moves to the pressure release position. This phase continues until the angular wheel deceleration becomes positive (Fig. 2), which is slightly different from the condition used in the Bosch ABS cycle. In phase 4, the brake pressure is held constant for a specified time duration (also referred to as the apply delay) or until the angular wheel acceleration exceeds the pronounced threshold value $(+A)$. In phase 5 , the brake pressure continues to increase for as long as the wheel's peripheral acceleration remains above the threshold $(+\mathrm{A})$. In phase 6 , the brake pressure is again held constant for a specified time duration or until the angular wheel acceleration drops below the acceleration threshold $(+a)$. In phase 7 , the brake pressure is built up in stages (using an adjustable duty cycle) as the solenoid valve applies a stepwise pressure increase. In this stage greater braking performance is achieved close to peak friction, while minimizing the wheel lock-up potential. This phase continues until the wheel's peripheral deceleration exceeds the threshold $(-a)$ again. In phase 8 , the brake cycle returns to phase 3 , without waiting for the threshold $\lambda_{1}$, and finally a new control cycle starts.

\section{TYRE MODELS}

To study the influence of tyre model transience on ABS braking, three different transient tyre models, based on the single-point contact approach [15], are used. Apart from the representation of tyre carcass compliances 
and contact patch slip properties, these various transient tyre models also differ in the way the relaxation length is considered. The following sections present a brief description of the chosen transient tyre models.

\subsection{Stretched-string tyre model}

In this tyre model, the first-order differential equation, representing the relaxation length concept, is used as a central approach to incorporate the transient tyre behaviour. The first-order relaxation length accounts for the carcass compliance with respect to the wheel rim in both longitudinal and lateral directions Adopted from the work of Pacejka [15], the model follows a simplified form of the string method, originally developed to study the tyre shimmy motion by Pacejka [7]. This approach is also used in the PAC 2002 version of the magic tyre formula [6]. In its simplest form, the string model reduces to a single-contact-point tyre model, whereby the contact patch is connected to the rim by a longitudinal and lateral spring, representing the stiffness of the carcass in the respective directions. In turn, the contact patch is able to slide with respect to the road in both lateral and longitudinal directions. Figure 3 shows an arbitrary operating position of the tyre indicating the slip conditions, as well as the lateral and longitudinal deflection of the carcass.

The rate of change of lateral deflection, $v_{1}$, between the contact point and the rim can be expressed using the following differential equation

$$
\sigma_{\alpha} \frac{\mathrm{d} \nu_{1}}{\mathrm{~d} t}+\left|U_{\mathrm{w}}\right| \nu_{1}=\sigma_{\alpha} V_{\mathrm{s} y}
$$

Similarly, the deflection in the longitudinal direction can be expressed as

$$
\sigma_{\kappa} \frac{\mathrm{d} u_{1}}{\mathrm{~d} t}+\left|U_{\mathrm{w}}\right| u_{1}=-\sigma_{\kappa} V_{\mathrm{s} x}
$$

Both longitudinal and lateral relaxation lengths are defined in terms of vertical load as [19]

$$
\begin{aligned}
\sigma_{\kappa}= & F_{z}\left(p_{\mathrm{T} x 1}+p_{\mathrm{T} x 2} \mathrm{~d} f_{z}\right) \exp \left(p_{\mathrm{T} x 3} \mathrm{~d} f_{z}\right)\left(\frac{r_{0}}{F_{z 0}}\right) \\
\sigma_{\alpha}= & p_{\mathrm{T} y 1} F_{z 0} \sin \left\{2 \arctan \left[\frac{F_{z}}{\left(p_{\mathrm{T} y 2} F_{z 0}\right)}\right]\right\} \\
& \times\left(1-p_{\mathrm{K} y 3}\left|\gamma_{y}\right|\right) r_{0}
\end{aligned}
$$

The transient value of longitudinal slip and the slip angle, which are further used in the magic formula equations for forces and moment calculation, can be expressed as (based on the string deformation)

$$
\begin{aligned}
\kappa^{\prime} & =\frac{u_{1}}{\sigma_{\kappa}} \operatorname{sign}\left(U_{\mathrm{w}}\right) \\
\alpha^{\prime} & =\operatorname{atan}\left(\frac{v_{1}}{\sigma_{\alpha}}\right)
\end{aligned}
$$

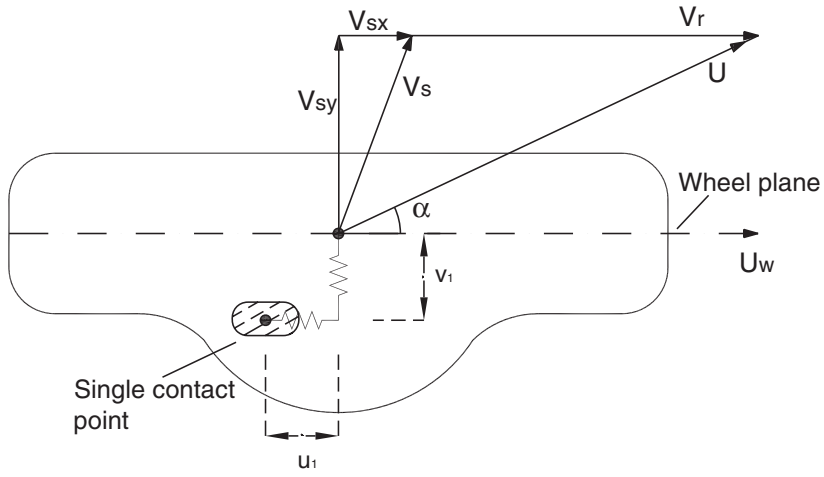

Fig. 3 Lateral and longitudinal carcass deflection of the single-point contact tyre model

\subsection{Modified stretched-string tyre model}

In the previous stretched-string model, the relaxation length is expressed as a variable of vertical load, without taking tyre slip into account. This means the relaxation length does not change with an increasing slip angle, unlike what happens in practice. To overcome the limitations of the simple stretched-string model, Pacejka [15] suggested a stretched-string model with an elastic tread element.

The first-order relaxation length equation in this model is similar to the stretched-string model (equation (7)), with the relaxation length $\sigma_{\alpha}$ replaced by $\sigma_{\alpha}^{*}$

$$
\frac{\mathrm{d} v_{1}}{\mathrm{~d} t}+\frac{1}{\sigma_{\alpha}^{*}}\left|U_{\mathrm{w}}\right| \nu_{1}=\left|U_{\mathrm{w}}\right| \alpha=-V_{\mathrm{s} y}
$$

The relaxation length in this model is determined by considering the transient effects of variation in vertical load as well as slip. The $\sigma_{\alpha}^{*}$ can be represented as [15]

$$
\sigma_{\alpha}^{*}=\frac{1}{C_{F y}} \frac{F_{y}}{\tan \alpha^{\prime}}=\frac{\sigma_{\alpha \mathrm{o}}}{C_{F \alpha}} \frac{F_{y}}{\tan \alpha^{\prime}} \approx \frac{\sigma_{\alpha \mathrm{o}}}{C_{F \alpha}} \frac{\left|F_{y}^{\prime}\right|+C_{F \alpha} \varepsilon_{\mathrm{F}}}{\left|\tan \alpha_{\mathrm{f}}^{\prime}\right|+\varepsilon_{\mathrm{F}}}
$$

where $\sigma_{\alpha \mathrm{o}}$ denotes the initial relaxation length at $\alpha=0$, which is given as

$$
\sigma_{\alpha \mathrm{o}}=\frac{C_{F \alpha}}{C_{F y}}
$$

Similar to the lateral relaxation model, the firstorder differential equation for the longitudinal deflection can be represented as

$$
\frac{\mathrm{d} u_{1}}{\mathrm{~d} t}+\frac{1}{\sigma_{\kappa}^{*}}\left|U_{\mathrm{w}}\right| u_{1}=\left|U_{\mathrm{w}}\right| \kappa=-V_{\mathrm{s} x}
$$

where the longitudinal relaxation length $\sigma_{\kappa}^{*}$ can be obtained using the following expression

$$
\sigma_{\kappa}^{*}=\frac{1}{C_{F x}} \frac{F_{x}}{\kappa^{\prime}}=\frac{\sigma_{\kappa \mathrm{o}}}{C_{F \kappa}} \frac{F_{x}}{\kappa^{\prime}} \approx \frac{\sigma_{\kappa \mathrm{o}}}{C_{F \kappa}} \frac{\left|F_{x}^{\prime}\right|+C_{F \kappa} \varepsilon_{\mathrm{F}}}{\left|\kappa^{\prime}\right|+\varepsilon_{\mathrm{F}}}
$$


The term $\sigma_{\kappa \mathrm{o}}$ represents the initial relaxation length at $\kappa^{\prime}=0$, which is given as

$$
\sigma_{\kappa \mathrm{o}}=\frac{C_{F \kappa}}{C_{F x}}
$$

The transient lateral slip $\tan \alpha^{\prime}$ can be computed from the lateral deflection $v_{1}$ through

$$
\tan \alpha^{\prime}=\frac{v_{1}}{\sigma_{\alpha}^{*}}
$$

Similarly, the transient slip can also be computed from the longitudinal deflection $u_{1}$ through

$$
\kappa^{\prime}=\frac{u_{1}}{\sigma_{\kappa}^{*}}
$$

The magic formula equations, based on the transient slip quantities $\kappa^{\prime}$ and $\alpha^{\prime}$, can be evaluated to calculate the transient forces and moments as

$$
\begin{aligned}
& F_{x}=F_{x}\left(\alpha^{\prime}, \kappa^{\prime}, F_{z}\right) \\
& F_{y}=F_{y}\left(\alpha^{\prime}, \kappa^{\prime}, \gamma, F_{z}\right) \\
& M_{z}^{\prime}=M_{z}^{\prime}\left(\alpha^{\prime}, \kappa^{\prime}, \gamma, F_{z}\right)
\end{aligned}
$$

\subsection{Contact mass transient tyre model}

The stretched-string models described in the previous sections are based on the relaxation length concept, which takes into account the combined effect of carcass compliance and contact patch slip properties and in the process accommodate the lag in the response to lateral and longitudinal slips. Since the response

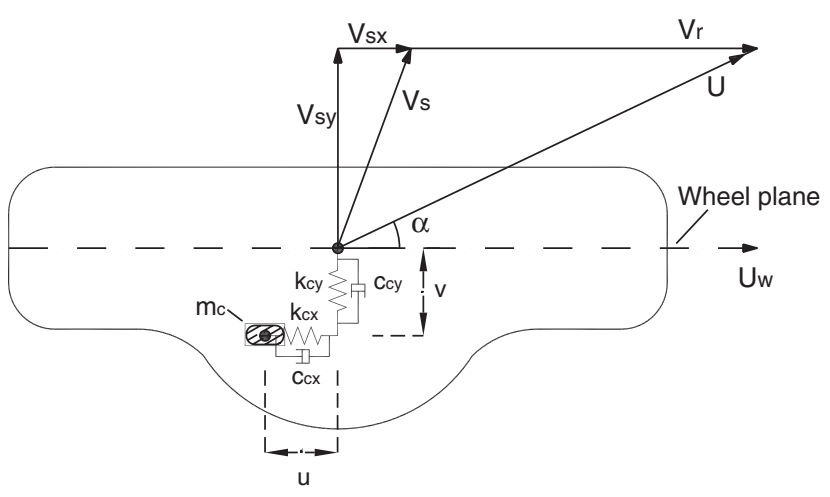

Fig. 4 Contact mass model with contact patch mass and carcass compliances

Table 1 Braking friction coefficients (for the vehicle speed of $20 \mathrm{~m} / \mathrm{s}$ )

\begin{tabular}{ll}
\hline Road surface & $\begin{array}{l}\text { Brake friction } \\
\text { coefficients }\end{array}$ \\
\hline Dry asphaltic concrete & 0.85 \\
Wet asphaltic concrete & 0.5 \\
Dry packed snow & 0.25 \\
\hline
\end{tabular}

lag is dependent on the vertical load and the wheel slip variations, the relaxation length in the non-linear stretched-string model reduces at a higher level of slip. However, this approach is not numerically very stable. Furthermore, the situation at combined slip can become somewhat cumbersome. To overcome this problem, a slightly different approach can be followed, based on the separation of the carcass compliance and contact slip properties by explicitly incorporating carcass springs in the model. In the current model, the contact patch is defined with inertia such that it can deflect in the circumferential and lateral directions with respect to the lower part of the rim. Here, the carcass compliance together with the slip model of the contact patch automatically takes care of the vertical load-dependent lag, in addition to a decrease in lag with an increasing slip. This approach was initially employed by Pacejka and Besselink [20], based on an earlier work by van der Jagt et al. [8] in their 1997 version of the magic formula transient tyre model, which was later incorporated in the PAC 2002 tyre model [6].

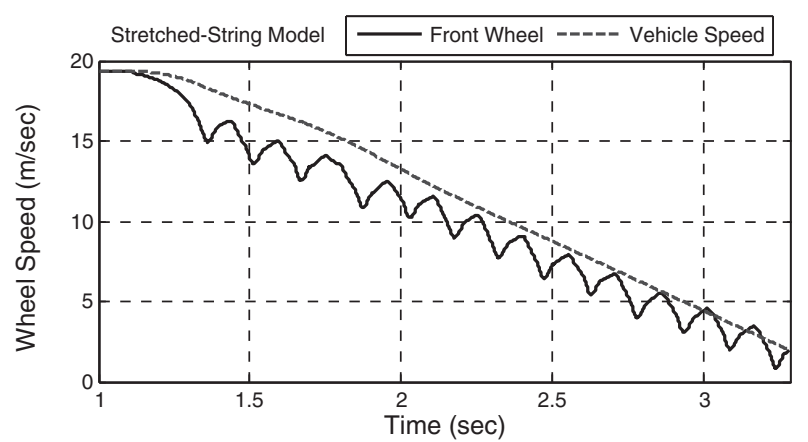

(a)

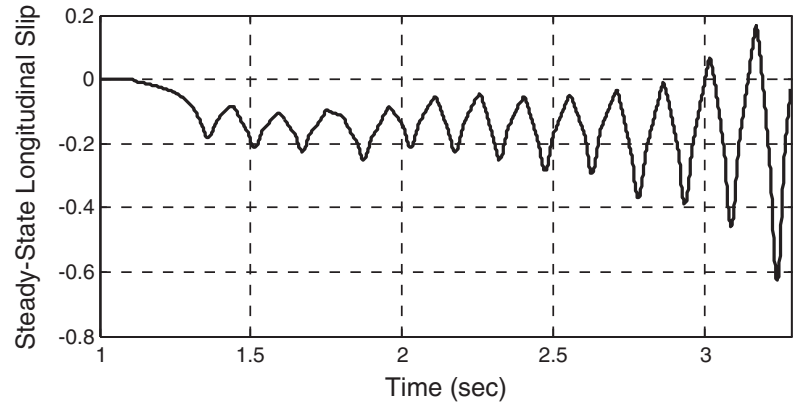

(b)

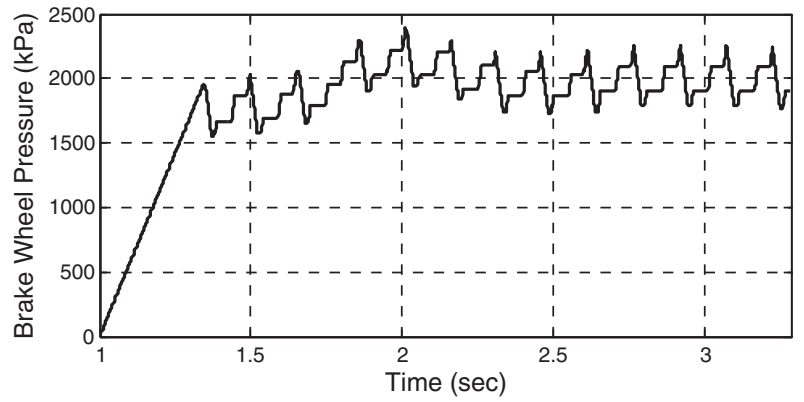

(c)

Fig. 5 Straight-line braking with stretched-string model $(V=70 \mathrm{~km} / \mathrm{h}$, dry $)$ 
By incorporating the contact patch mass ' $m_{\mathrm{c}}$ ', the carcass stiffness ' $k_{\mathrm{c} x, y}$ ', and damping ratios ' $c_{\mathrm{c} x, y}$ ' (see Fig. 4), the equations of motion for the contact can be represented in the following form (employing one DOF model for the single point mass)

$$
\begin{aligned}
& m_{\mathrm{c}} \dot{V}_{\mathrm{s} x}^{*}+c_{\mathrm{c} x} \dot{u}+k_{\mathrm{c} x} u=F_{x}\left(\kappa^{\prime}, \alpha^{\prime}, F_{z}\right) \\
& m_{\mathrm{c}} \dot{V}_{\mathrm{s} y}^{*}+c_{\mathrm{c} y} \dot{v}+k_{\mathrm{c} y} v=F_{y}\left(\alpha^{\prime}, \kappa^{\prime}, \gamma, F_{z}\right)
\end{aligned}
$$

In addition, the contact mass model also includes a simple relaxation length model to enable computations at vanishing speeds

$$
\begin{aligned}
& \sigma_{\mathrm{c}} \frac{\mathrm{d} \kappa^{\prime}}{\mathrm{d} t}+\left|U_{\mathrm{w}}\right| \kappa^{\prime}=-V_{\mathrm{s} x}^{*} \\
& \sigma_{\mathrm{c}} \frac{\mathrm{d} \alpha^{\prime}}{\mathrm{d} t}+\left|V_{\mathrm{w}}\right| \alpha^{\prime}=-V_{\mathrm{s} y}^{*}
\end{aligned}
$$

From these equations the transient slip quantities $\kappa^{\prime}, \alpha^{\prime}$ are obtained, which act as an input in the steadystate slip force model (right-hand side of equations (24) and (25)). The contact relaxation length $\sigma_{\mathrm{c}}$ is given a small value such as half the contact length.

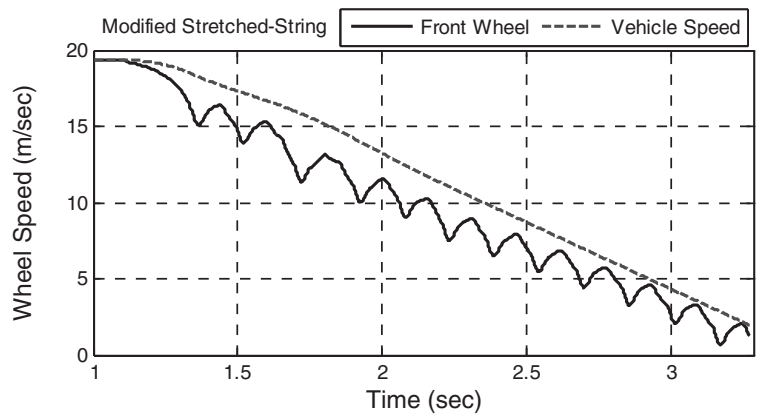

(a)

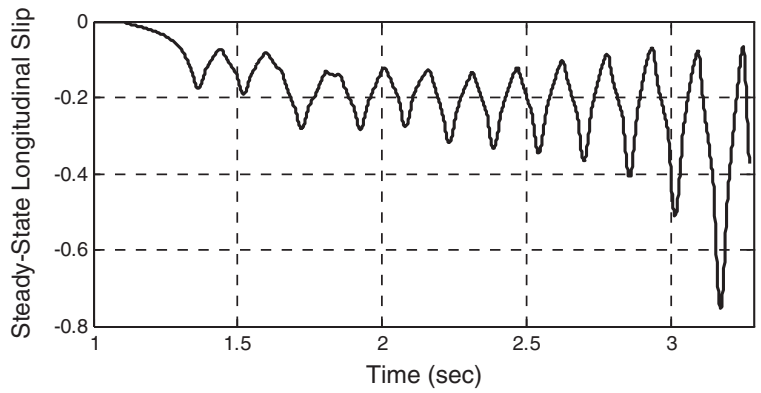

(c)

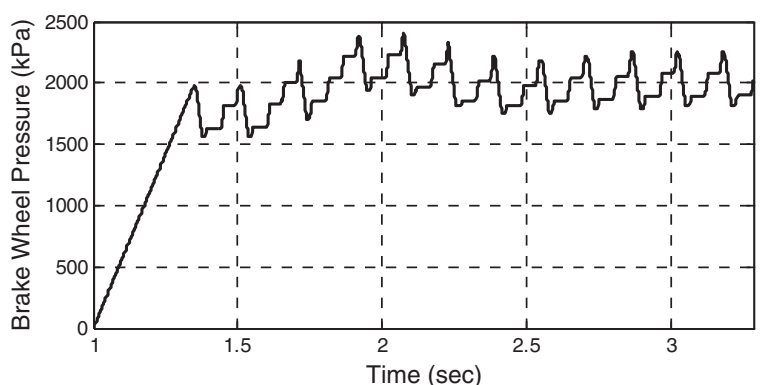

(e)
The longitudinal and lateral deflection rates required in equations (24) and (25) can be computed by the difference in the slip velocities

$$
\begin{aligned}
& \dot{u}=V_{\mathrm{s} x}^{*}-V_{\mathrm{s} x} \\
& \dot{v}=V_{\mathrm{s} y}^{*}-V_{\mathrm{s} y}
\end{aligned}
$$

where $V_{\mathrm{s} x}, V_{\mathrm{s} y}$ denote the wheel slip velocity of the slip point, which is attached to the wheel rim at distance $r_{\mathrm{e}}$ (effective rolling radius) below the wheel centre in the wheel centre plane. The $V_{\mathrm{s} x}$ can be expressed as

$$
V_{\mathrm{s} x}=U_{\mathrm{w}}-r_{\mathrm{e}} \Omega
$$

The effective rolling radius $r_{\mathrm{e}}$ is expressed as a function of vertical load on the wheel based on the load range of operation [15]. $U_{\mathrm{w}}$ denotes the longitudinal wheel velocity (represented in terms of wheel coordinates).

The lateral slip velocity $V_{\text {sy }}$ can be equated to the lateral wheel velocity $V_{\mathrm{w}}$ (represented in terms of wheel

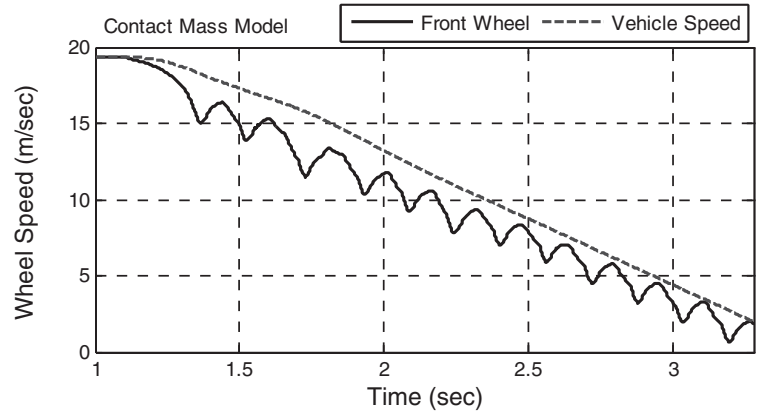

(b)

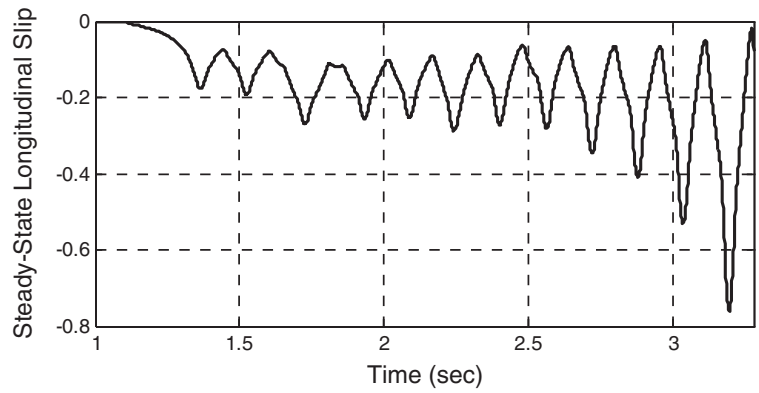

(d)

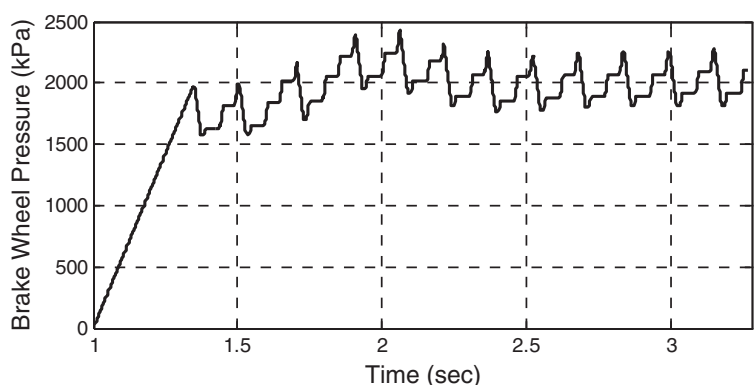

(f)

Fig. 6 Straight-line braking with modified stretched-string and contact mass models $(V=70 \mathrm{~km} / \mathrm{h}$, dry) 
coordinates)

$$
V_{\mathrm{sy}}=V_{\mathrm{w}}
$$

The tyre longitudinal and lateral slip can be expressed as

$$
\begin{aligned}
& \kappa=-\frac{V_{\mathrm{s} x}}{\left|U_{\mathrm{w}}\right|} \\
& \tan \alpha=\frac{V_{\mathrm{s} y}}{\left|U_{\mathrm{w}}\right|}
\end{aligned}
$$

Here, equations (30) to (33) are generic relations employed in the vehicle model for the calculation of slip quantities.

Once the longitudinal and lateral deflections are obtained by solving equations (24) and (25), the longitudinal and lateral forces acting on the wheel rim, finally, become

$$
\begin{aligned}
& F_{x a}=c_{\mathrm{c} x} \dot{u}+k_{\mathrm{c} x} u \\
& F_{y a}=c_{\mathrm{c} y} \dot{v}+k_{\mathrm{c} y} v
\end{aligned}
$$

\section{ABS SIMULATION}

During a critical manoeuvre, the response behaviour of a vehicle, while being intervened by an active control system such as ABS, is affected by the rapid and fast changing tyre oscillations that occur due to the brake torque variations and the unevenness of the road surface. The transient influence of a tyre becomes crucial during such operations. The interaction of tyre transience with the anti-lock braking response is studied here, applying the earlier described transient tyre models and the ABS system. The ABS simulation is carried out for straight-line braking, as well as braking during cornering on various surfaces. In addition, the responses of different tyre models to step variations in brake pressure is analysed. The road-tyre friction for different surfaces is modelled, using the scale factor for the peak friction coefficients, such that the peak factor ' $D$ ' in the magic tyre formula is modified to generate a peak force corresponding to the coefficient of friction under static cornering load (see Table 1).

The brake force coefficient for a road surface varies with longitudinal slip. This has a strong dependence on road condition (texture, construction, temperature, and contamination) along with the vehicle speed and the tyre condition, as shown in a study by Harned et al. [21]. However, a constant value is selected here (Table 1) for a particular type of road surface, which is in relation to the vehicle speed used in the various manoeuvres.

\subsection{Straight-line braking}

The results for straight-line braking are presented in this section by performing a comparative evaluation of the three transient tyre models on the above different surfaces. In the straight-line braking simulation, the initial vehicle speed was kept at $70 \mathrm{~km} / \mathrm{h}(19.4 \mathrm{~m} / \mathrm{s})$ and brake was applied after a second. Figure 5 shows the simulation results for the stretched-string tyre model on the dry surface, where only the front wheel plots are displayed. The peripheral wheel speed plot is shown in Fig. 5(a) along with the vehicle longitudinal speed projected at each wheel. At lower speeds, the wheel speed fluctuates about the vehicle speed, during the brake release phase. This behaviour is also visible from the brake slip plot of Fig. 5(b), where the slip becomes momentarily positive. As the relaxation length in the stretched-string tyre model does not vary with slip, this means that towards the end of simulation the relaxation length still stays rather high, when compared with what would happen in practice. This eventually leads to a reduction in the effective damping in the contact and promotion of oscillatory behaviour at lower speeds.

In comparison with the stretched-string tyre model, the modified stretched-string tyre model and the

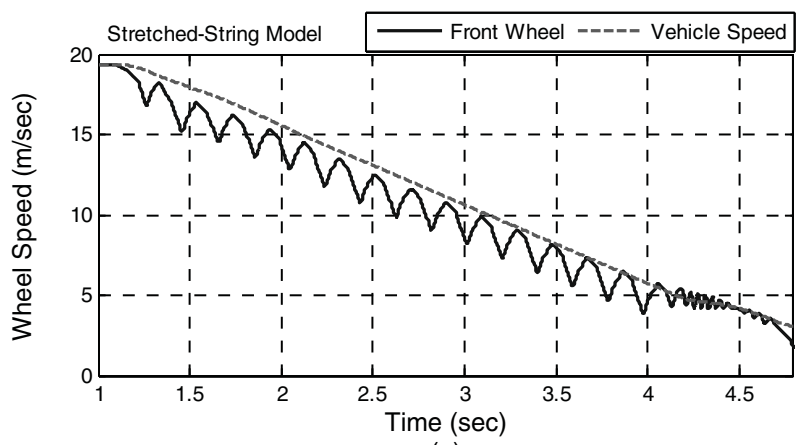

(a)

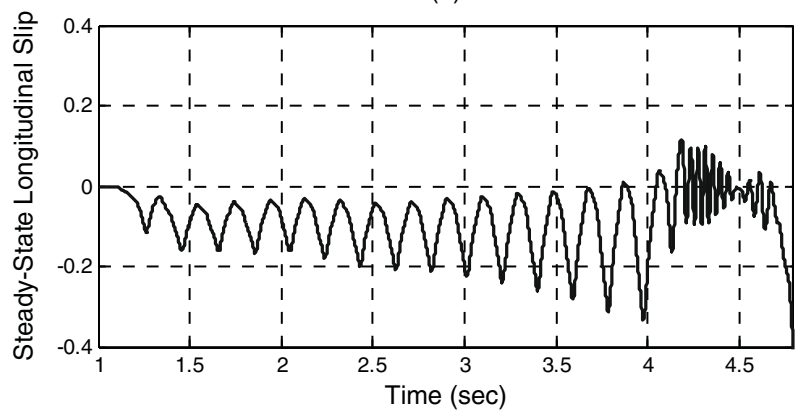

(b)

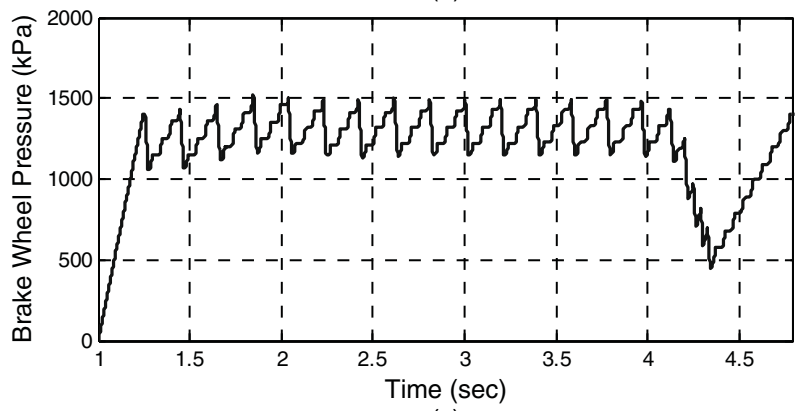

(c)

Fig. 7 Straight-line braking with stretched-string model ( $V=70 \mathrm{~km} / \mathrm{h}$, wet) 
contact mass model results demonstrate smooth and well-behaved ABS cycle, as shown in the response plots of wheel speed, steady-state longitudinal slip, and brake wheel pressure in Fig. 6. During the ABS operation, the correct representation of the transient slip becomes crucial, particularly at low wheel speeds. As the response lag in both these tyre models take into account the variations in wheel slip along with the vertical load, the results represent more realistic performance.

Similar to the straight-line braking on a dry surface, the ABS simulation results on a wet surface are compared for the three different tyre models. Figure 7 shows the simulation results for the stretched-string tyre model on the wet surface, where only the front wheel plots are displayed. Again the result highlights the undesirable behaviour of the stretched-string tyre model at low vehicle speeds. As evident from the plots of wheel speed (Fig. 7(a)) and the longitudinal slip (Fig. 7(b)), the wheel speed fluctuates about the vehicle speed. As the friction force saturates earlier on a wet surface, a slight release of brake pressure accelerates

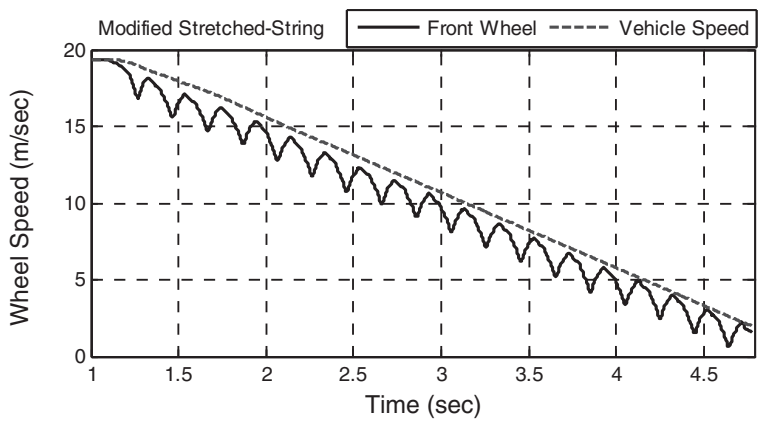

(a)

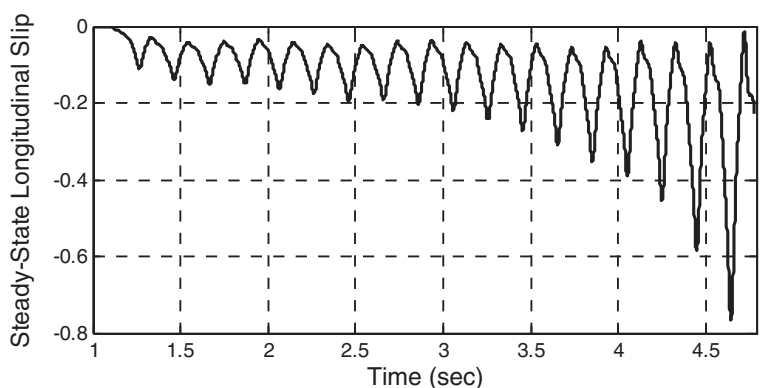

(c)

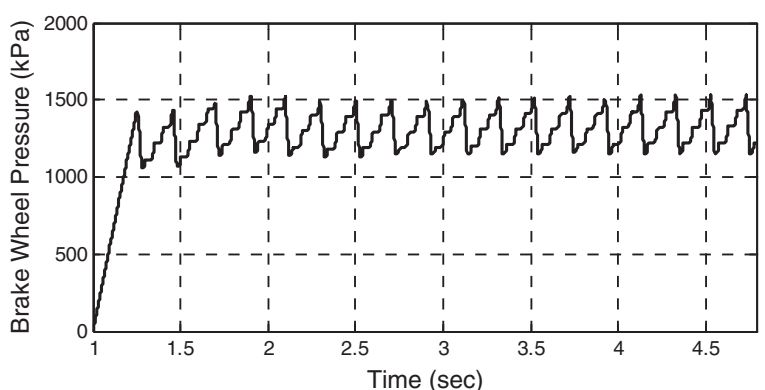

(e) the wheel very rapidly. As the relaxation length is still higher for the stretched-string tyre model, the tyre response lag remains larger, which produces a more oscillatory behaviour.

In contrast to the stretched-string tyre model, both the modified stretched string and the contact mass models demonstrate well-behaved ABS cycles, shown in Fig. 8 (left- and right-hand-side plots, respectively). The brake pressure profile in both cases is quite repetitive and smooth throughout the simulations, which to a certain extent also highlights the fact that external disturbances such as road irregularities were not considered in the ABS model. The ABS parameters used for determining the brake release, hold, and apply phases were kept the same for all the tyre models. Hence, similar brake pressure profiles can be observed for all the three models, until $4.2 \mathrm{~s}$, where the stretched-string tyre model starts to exhibit undesirable responses.

The results of straight-line braking for a dry snowy surface are also compared for the three tyre models. Figure 9 shows the results for the stretched-string transient tyre model (front wheel plots only), where

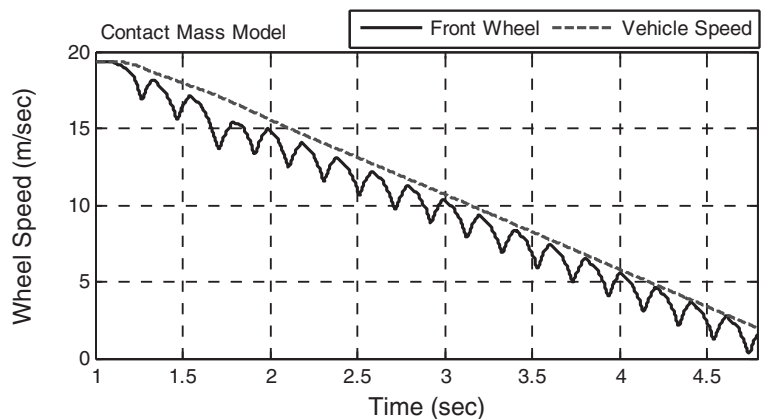

(b)

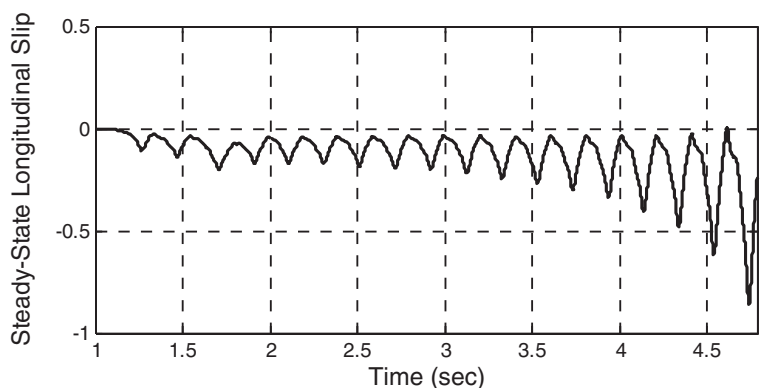

(d)

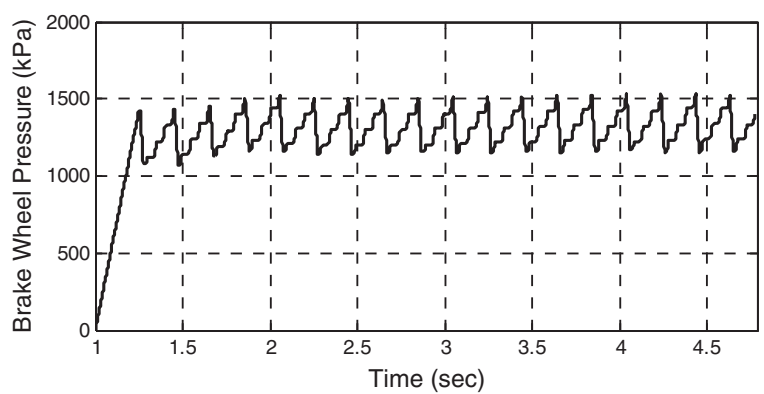

(f)

Fig. 8 Straight-line braking with modified stretched-string and contact mass models $(V=70 \mathrm{~km} / \mathrm{h}$, wet $)$ 


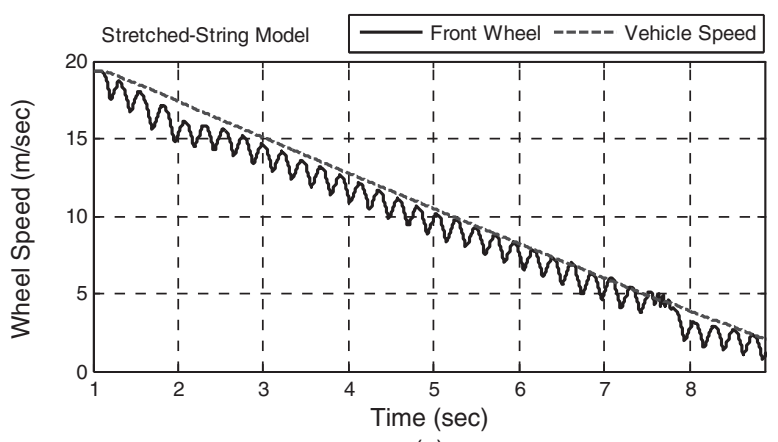

(a)

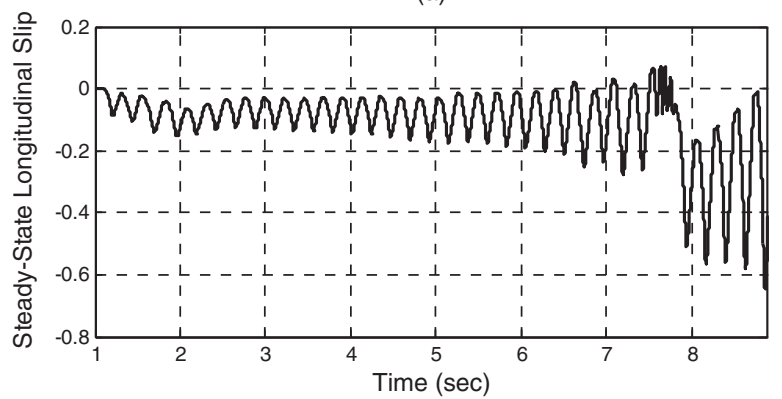

(b)

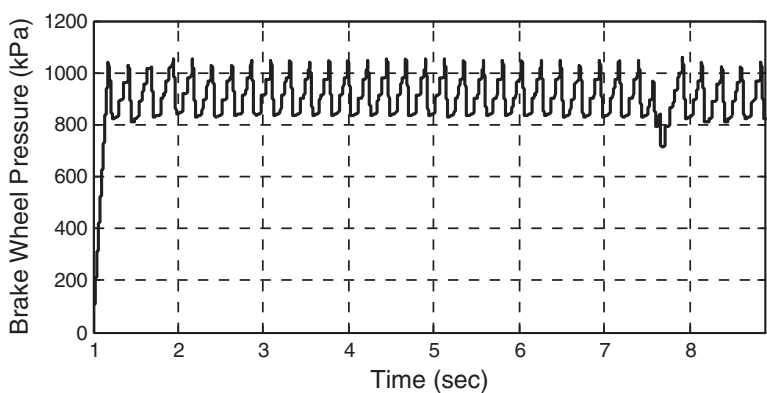

(c)

Fig. 9 Straight-line braking with stretched-string model $(V=70 \mathrm{~km} / \mathrm{h}$, snow)

it is evident that at lower speeds it starts exhibiting undesirable behaviour, which again highlights its inability to provide realistic contact damping, thus failing to contribute towards rapid attenuation of brake force oscillations. When comparing the behaviour of stretched-string tyre model on three different surfaces, it can be observed that its performance deteriorates with reducing friction. On snow, the friction force saturates earlier and with it the relaxation length diminishes and the compliant behaviour of the carcass deteriorates. This is not captured by the stretchedstring model, which continues to exhibit a significant oscillatory behaviour related to the unrealistically high relaxation length value.

The ABS results for the modified stretched-string tyre and the contact mass models are shown in Fig. 10 (left- and right-hand-side plots, respectively). Similar to the dry and wet surfaces' results, those for the snowy surface are again well behaved for both the models.

Comparison of the vehicle braking distance during the straight-line manoeuvre on different surfaces is shown in Table 2. It is hard to differentiate between the braking distance for the modified stretched-string tyre and the contact mass models on all the three chosen surfaces, which is in line with the similarity in their responses as seen earlier in the simulation plots. The braking distance of the stretched-string tyre model is also quite close to the other two, though marginally with a longer distance on all the three surfaces. It is evident that for straight-line manoeuvre, the braking distance does not provide a very useful insight to judge the performance of the three tyre models.

\subsection{ABS braking while cornering}

Similar to the straight-line braking, the ABS simulation for braking while cornering is also conducted for the three transient tyre models. The initial vehicle speed was again kept at $70 \mathrm{~km} / \mathrm{h}(19.4 \mathrm{~m} / \mathrm{s})$, with a steering hand-wheel input of $90^{\circ}$ (counter-clockwise) applied at $0.2 \mathrm{~s}$, and once the vehicle moves into a left-side corner, braking is initiated after $1 \mathrm{~s}$. Figure 11 shows the results for the stretched-string tyre model, where the wheel speed and longitudinal slip for the individual wheels are shown with an offset. This is for the sake of clarity in the presentation. As shown in Fig. 11, the wheel speed and the longitudinal slip plots exhibit large and undesirable oscillations at low speeds. As the wheel speed fluctuates past the vehicle speed, the longitudinal slip (Fig. 11(b)) and the braking force (Fig. 11(d)) momentarily attain positive values. This undesirable fluctuations of longitudinal or brake slip at lower speed also lead to a sudden drop in brake output pressure, as evident from the outer front wheel plot (Fig. 11(c)). Here, only three curves appear in the brake pressure plot (Fig. 11(c)), as both the rear wheels are operated on select-low mode, where the same brake output pressure is used. In comparison with the straight-line braking on a dry surface, the oscillatory behaviour of the stretched-string model is more visible during the cornering manoeuvre.

Figure 12 shows the lateral slip and the lateral force plots for the stretched-string tyre model. In comparison with the longitudinal slip, the lateral slip is relatively well behaved for most part of the brake cycle. However, towards the end of simulation, the lateral slip plot exhibits an undesirable response pattern, which can be attributed to the fact that the friction at the front wheels saturates.

The results for the modified stretched-string tyre model and the contact mass model for braking while cornering are shown in Figs 13 to 16 . In comparison with the stretched-string tyre model, the ABS results for both these transient tyre cases are well behaved. As the vehicle is steered leftwards, the inner front tyre (front left) attains its maximum friction potential much earlier than the outer front tyre, and hence the brake pressure build-up is less for the inner front tyres, resulting in a reduced longitudinal braking force. Consequently, the lateral or cornering force also drops 


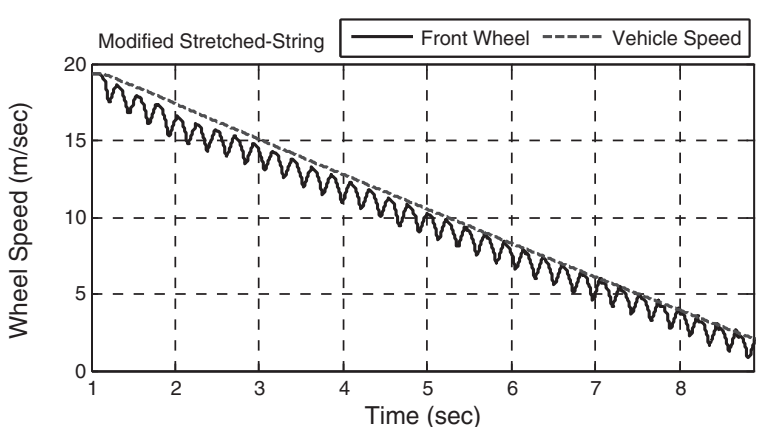

(a)

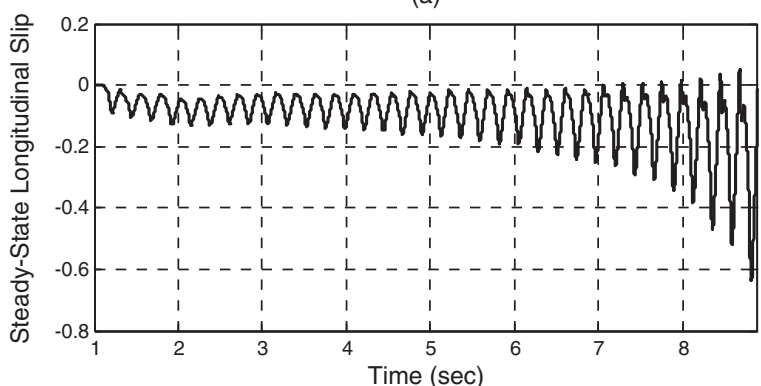

(c)

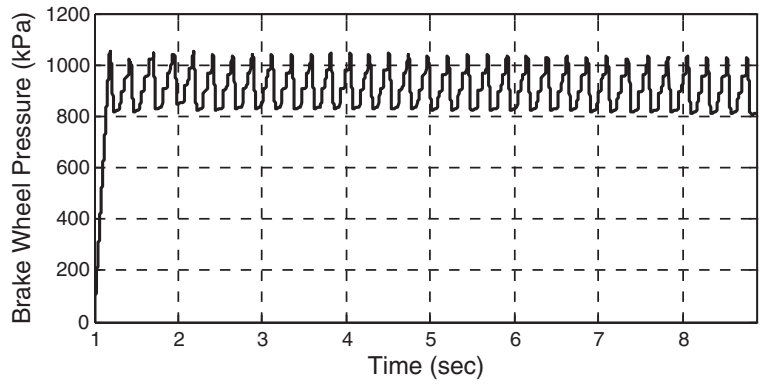

(e)

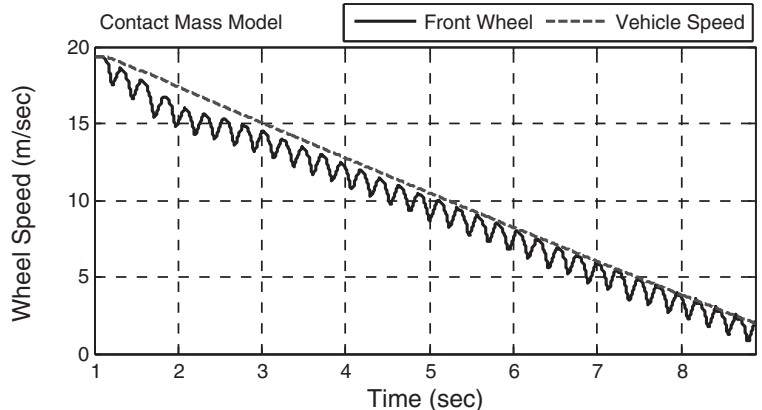

(b)

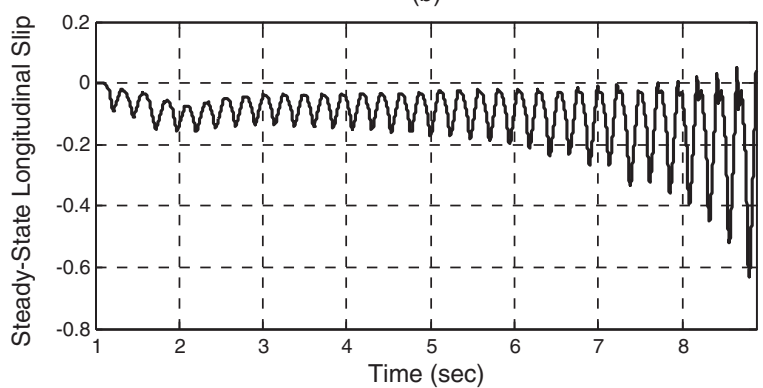

(d)

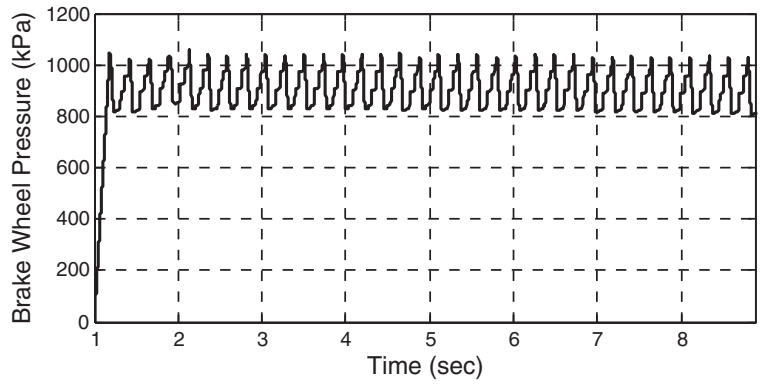

(f)

Fig. 10 Straight-line braking with modified stretched-string and contact mass models $(V=70 \mathrm{~km} / \mathrm{h}$, snow)

Table 2 Braking distance for the three transient tyre models during straight-line motion $(V=70 \mathrm{~km} / \mathrm{h})$

\begin{tabular}{llll}
\hline & \multicolumn{3}{c}{ Braking distance (m) } \\
\cline { 2 - 4 } Tyre models & Dry & Wet & \multirow{2}{*}{ Snow } \\
\hline Stretched-string transient tyre model & 25.82 & 41.33 & 82.83 \\
Modified stretched-string tyre model & 25.21 & 40.16 & 82.46 \\
Contact mass transient tyre model & 25.29 & 40.26 & 82.27 \\
\hline
\end{tabular}

for the inner front tyres. The plots of wheel speed, longitudinal and lateral slip, brake output pressure, and longitudinal and lateral force for both the tyre models show a similar pattern.

In the straight-line manoeuvre, the performance of the three transient tyre models was not very distinctive in terms of the braking distance. However, during the cornering manoeuvre, where more complicated interactions take place, the performance of the tyre models is more pronounced in terms of braking effectiveness. Figure 17 shows the vehicle paths for the three transient tyre models, where the vehicle furnished with the stretched-string model not only travels a longer distance, but also drifts slightly towards the inside of the turn. This behaviour can be further understood from the average braking force value at the individual wheels during the ABS cycle. As shown in Table 3, the average brake force at the front wheels is much lower for the stretched-string tyre model in comparison with the other two tyre models. The difference is noteworthy for the outer front wheel, where the brake force attains a positive value towards the end of simulation (Fig. 11(d)). This leads to a reduction in its brake effectiveness, resulting in a longer braking distance.

For the cornering manoeuvre, the performance of the three transient tyre models can be clearly evaluated from the above results, produced for the dry surface. A similar behaviour pattern was also seen for the wet and the snowy surfaces. Table 4 shows the braking distance comparison for the three transient tyre models on all the three surfaces, during the cornering manoeuvre. As evident, the braking distance in case of the stretched-string tyre model is consistently longer on all the three surfaces, in comparison with the 


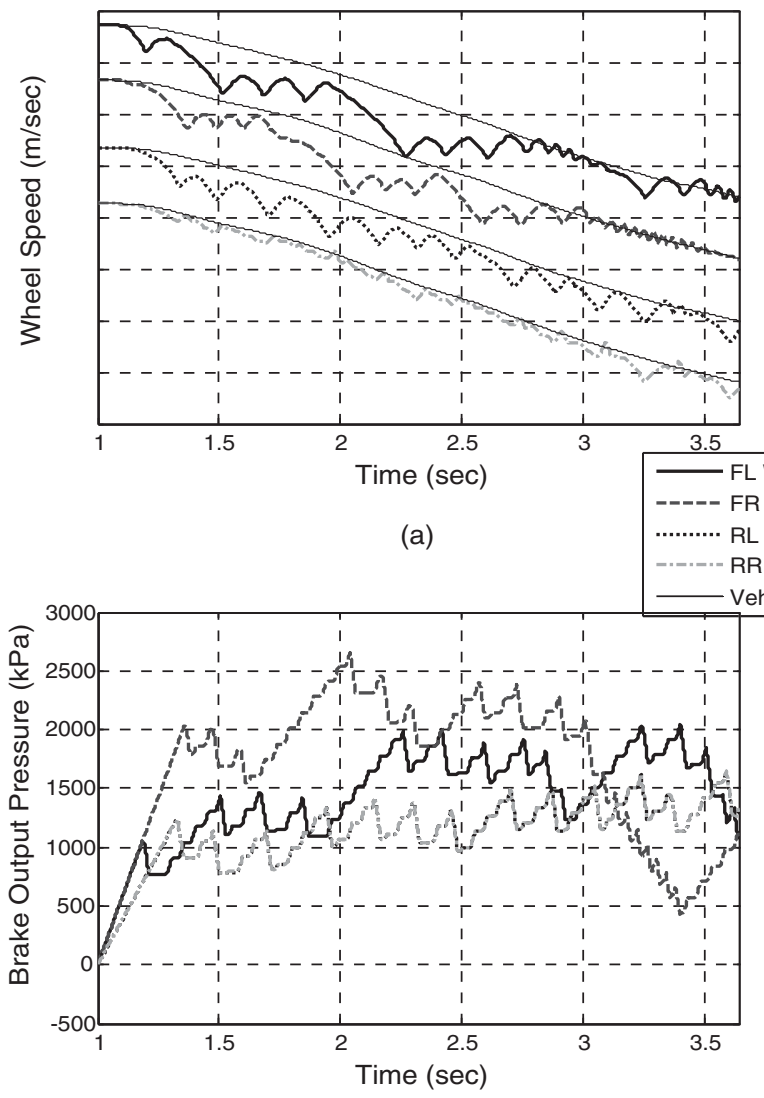

(c)

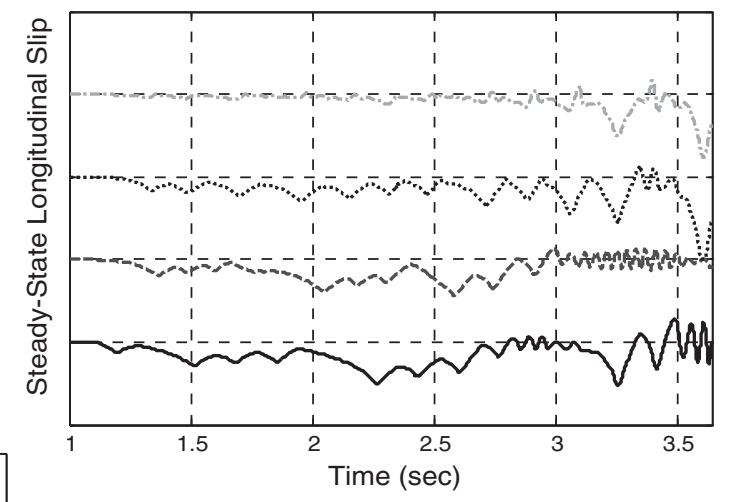

(b)

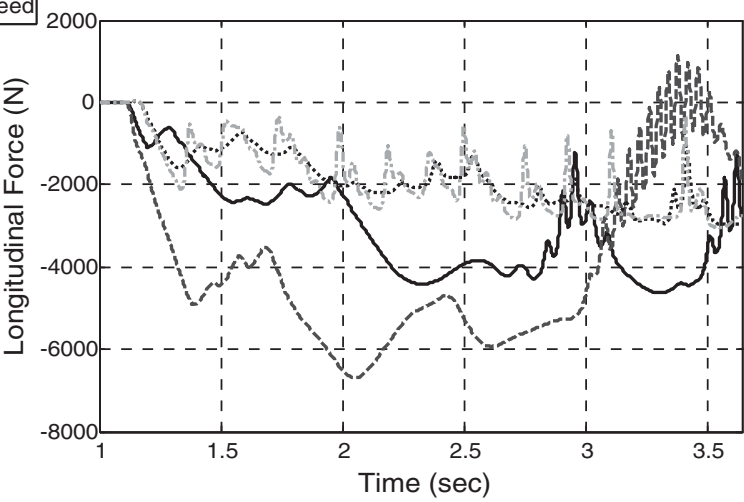

(d)

Fig. 11 Braking while cornering with the stretched-string model $(V=70 \mathrm{~km} / \mathrm{h}$, dry)
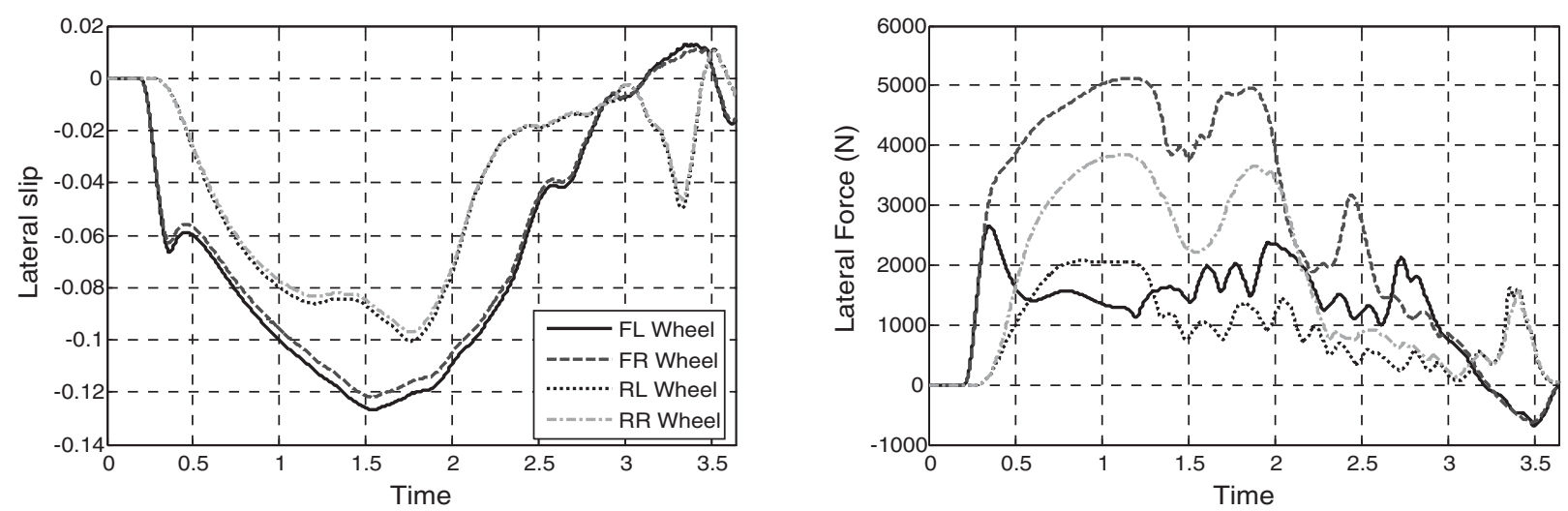

Fig. 12 Lateral slip/force plots with stretched-string model $(V=70 \mathrm{~km} / \mathrm{h}$, dry)

other two tyre models. The results reiterate the limitations of the stretched-sting tyre, in terms of brake effectiveness.

\subsection{Braking simulation with step variation in brake pressure}

The simulation results presented so far involved closed-loop ABS braking, where the influence of different transient tyre models was investigated. The analysis results clearly highlighted the limitations of the first-order relaxation length-based stretchedstring model, predicting the tyre behaviour in ABS applications. In comparison, both the modified stretched-string model and the contact mass model not only represented the tyre dynamic behaviour in a more realistic manner, but also produced similar results. The similarity in both these tyre models' results can be attributed to the fact that the current ABS braking model, while simulating the rapid brake pressure 

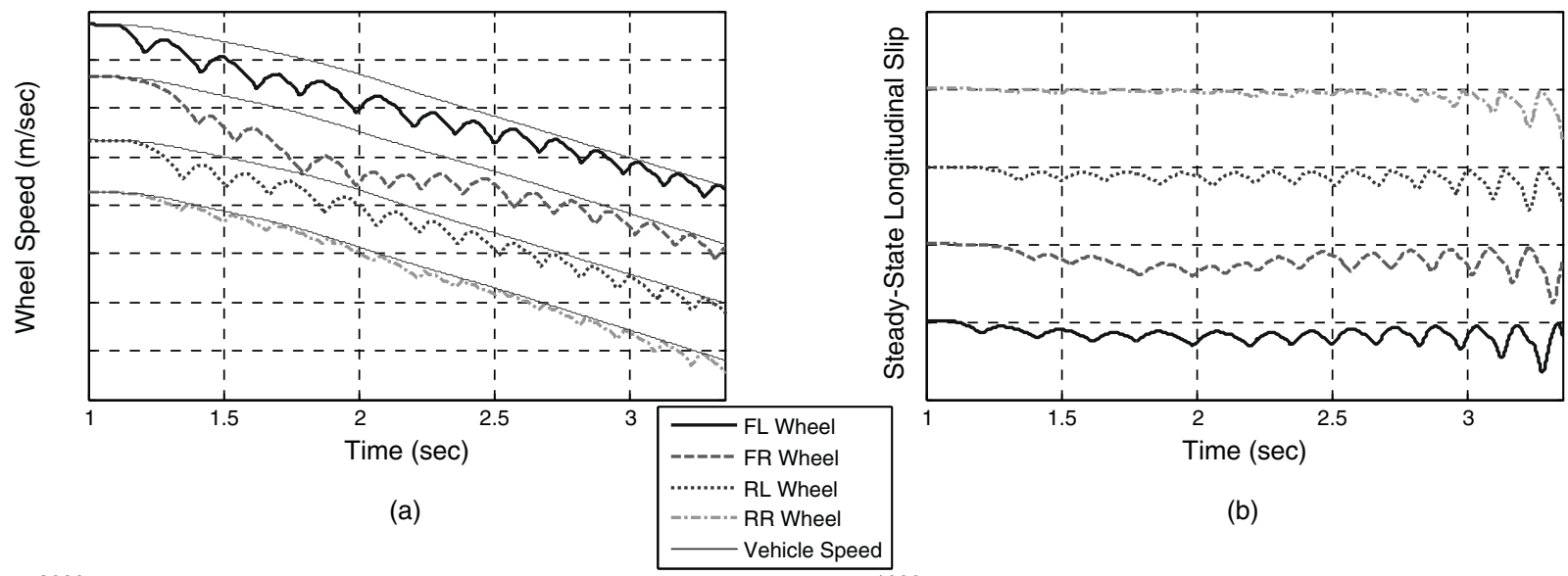

(b)

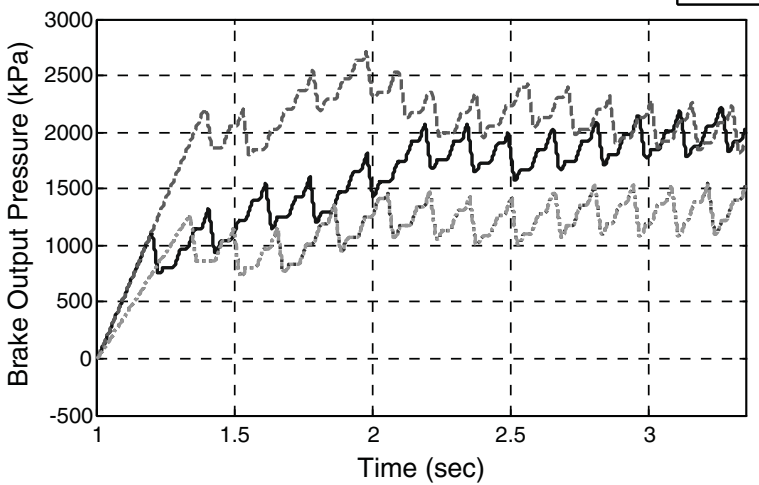

(c)

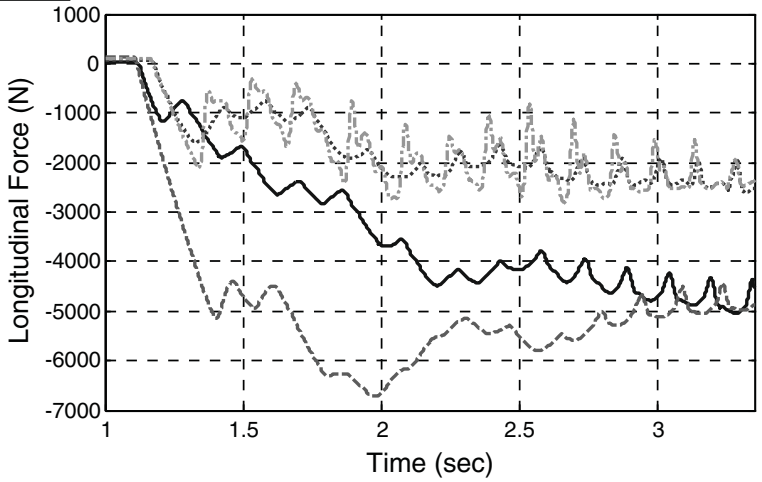

(d)

Fig. 13 Braking while cornering with modified stretched-string model $(V=70 \mathrm{~km} / \mathrm{h}$, dry)
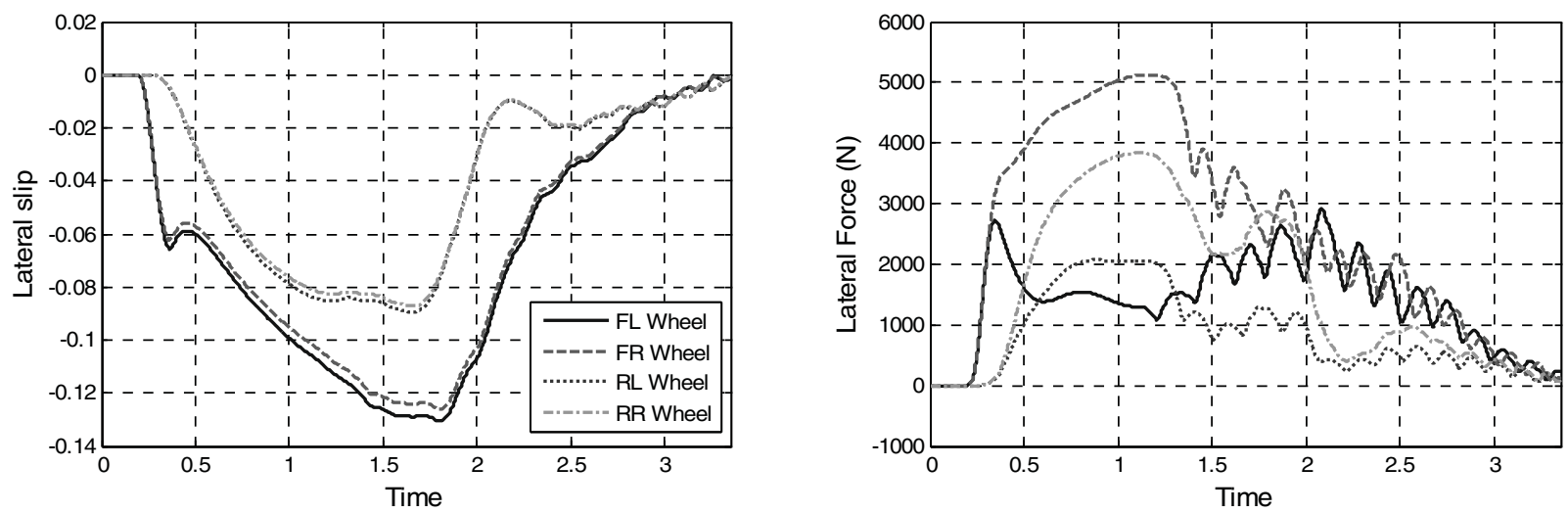

Fig. 14 Lateral slip/force plots with modified stretched-string model ( $V=70 \mathrm{~km} / \mathrm{h}$, dry)

build-up, hold, and reduction cycles (mostly repetitive in nature), tested only certain characteristics of a tyre model, namely the its ability to handle fast changing slip and wheel oscillations. In comparison, an ABS system in a production vehicle has to deal with a number of other transient events such as those originating from the brake modulator and the brake lines as an outcome of the transient pressure variations due to the brake fluid hydraulics and opening and closing of the valves, as well as irregularities of the road surface, etc. Consequently, the tyre in a production vehicle is exposed to more dynamic behaviour than what is simulated in the current ABS model.

Bearing these in mind, a new set of simulations are conducted, where a step brake pressure pulse is employed to excite the tyre transient behaviour. As opposed to the ABS simulation, the brake pressure in this case follows an open-loop cycle, where a pre-determined step brake pressure is applied to the wheels without any feedback to modulate the pressure cycle. Additionally, a short pressure pulse is added at the beginning of every pressure step so that the 


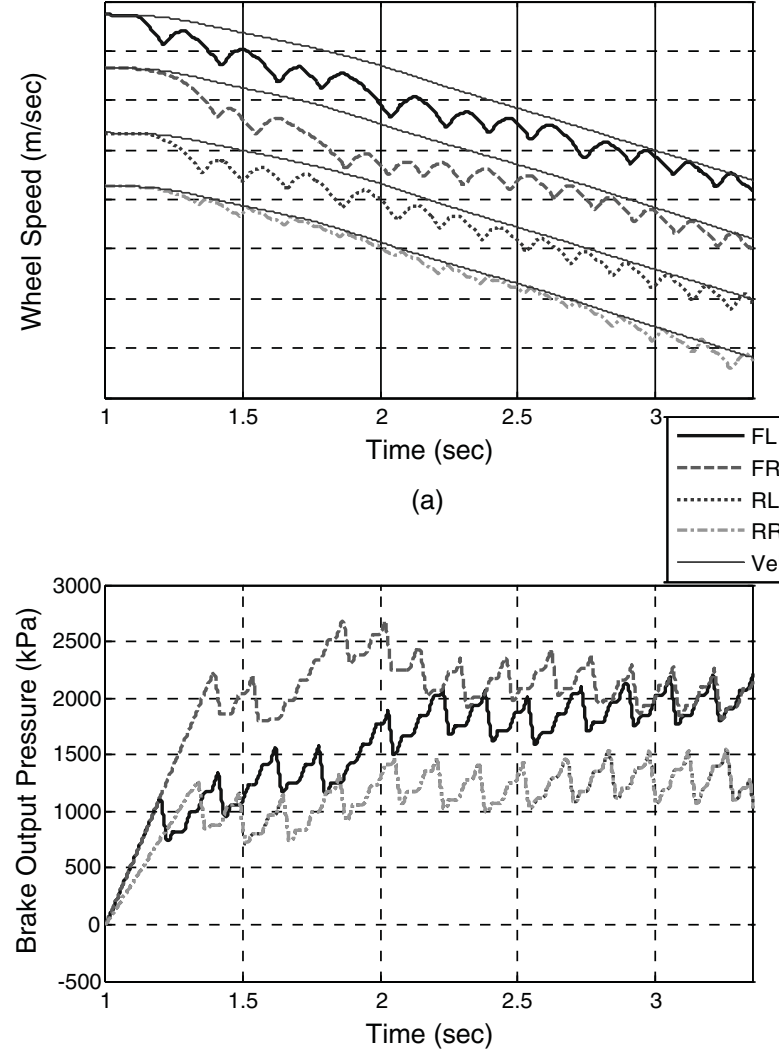

(c)

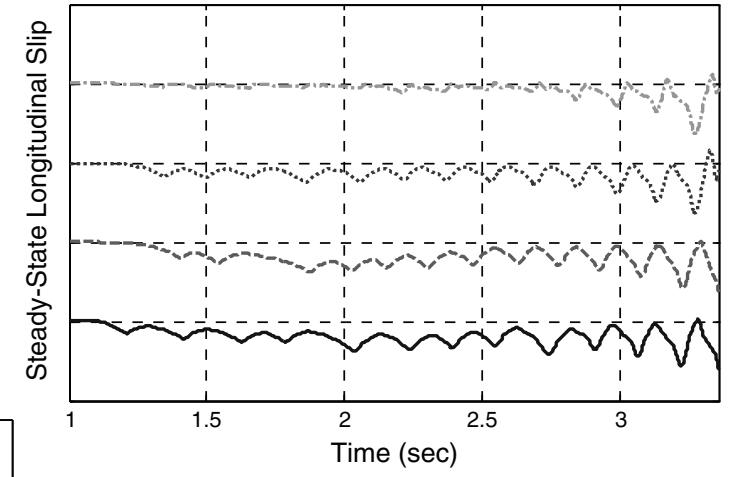

(b)

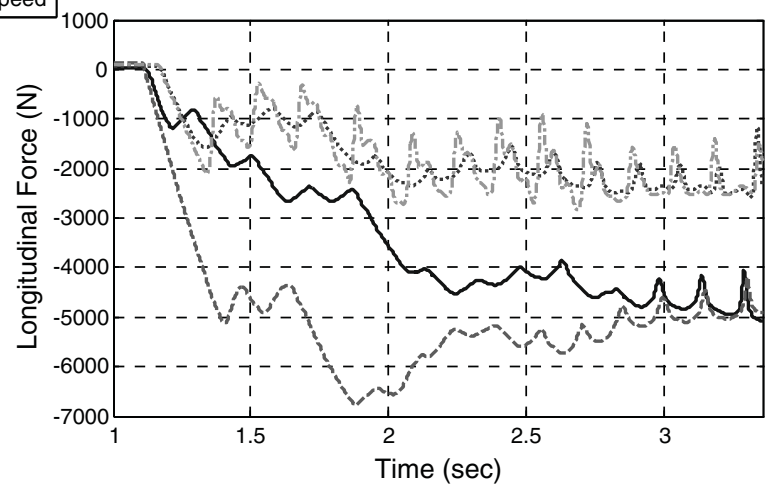

(d)

Fig. 15 Braking while cornering with contact mass model ( $V=70 \mathrm{~km} / \mathrm{h}$, dry)
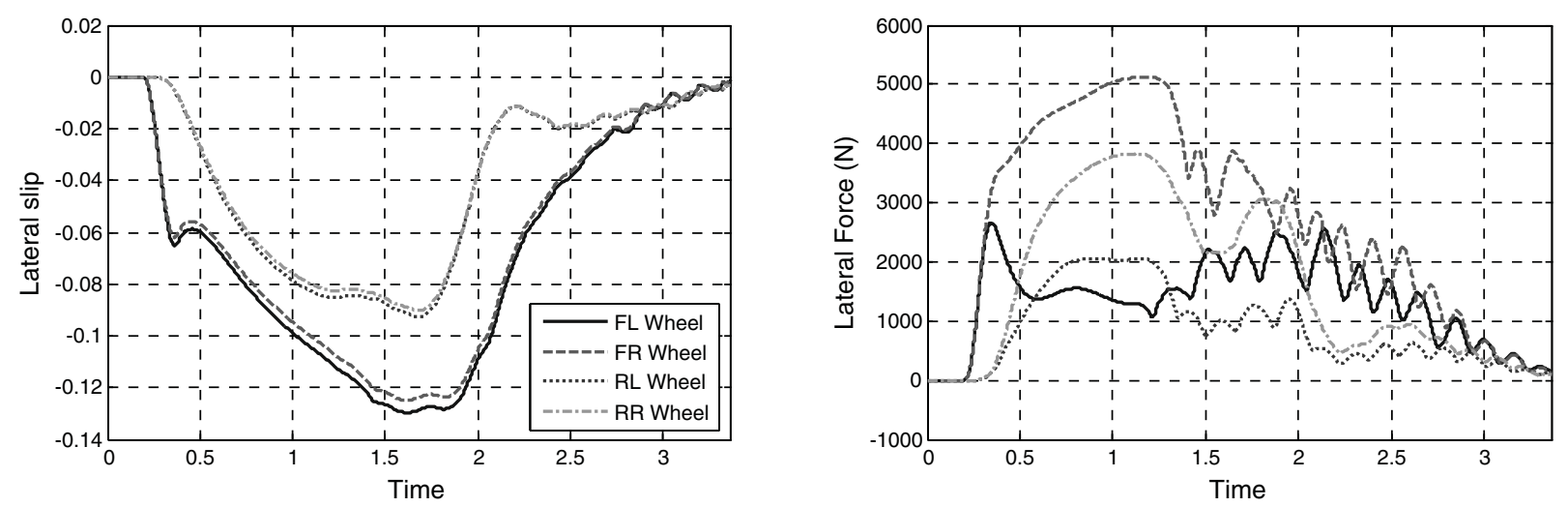

Fig. 16 Lateral slip/force plots with contact mass model $(V=70 \mathrm{~km} / \mathrm{h}$, dry)

dynamic behaviour of the transient tyre models can be studied further. In-line with the previous simulation runs, comparisons between the three transient tyre models are again made.

Figure 18 shows the step brake pressure variation plots for the three transient tyre models. The vehicle in this case is driven in a straight line with a constant speed of $34 \mathrm{~km} / \mathrm{h}$. A brake pressure with ramp input is initially applied to all the four wheels (front and rear distribution) at $0.5 \mathrm{~s}$, before the step brake input is initiated at $1.5 \mathrm{~s}$. The stepped brake pressure input with short pressure pulses is shown in Fig. 18(a), where the overall brake pressure gradually increases through the course of simulation, but the small step rise in brake pressure ensures that the vehicle decelerates gradually. As a result, the brake slip remains relatively low during the simulation with no danger of wheel locking. The interesting part of the simulation, however, is the tyre longitudinal force response to the excitation provided by the short pressure pulses.

Figure 18(b) shows the longitudinal force response for the stretched-string tyre model. It clearly shows that the force oscillations, following the excitations from the pressure pulses, are not damped quickly. 
These oscillations increase further, as the speed of the vehicle reduces. In comparison, the modified stretched-string tyre model shows better longitudinal force response (Fig. 18(c)), as the oscillations in this case are damped quicker. In the stretched-string and the modified stretched-string tyre models, the only source of damping is the friction at the contact patches. In the stretched-string tyre model, this damping mechanism is combined with the relaxation length, which stays constant in relation to slip.

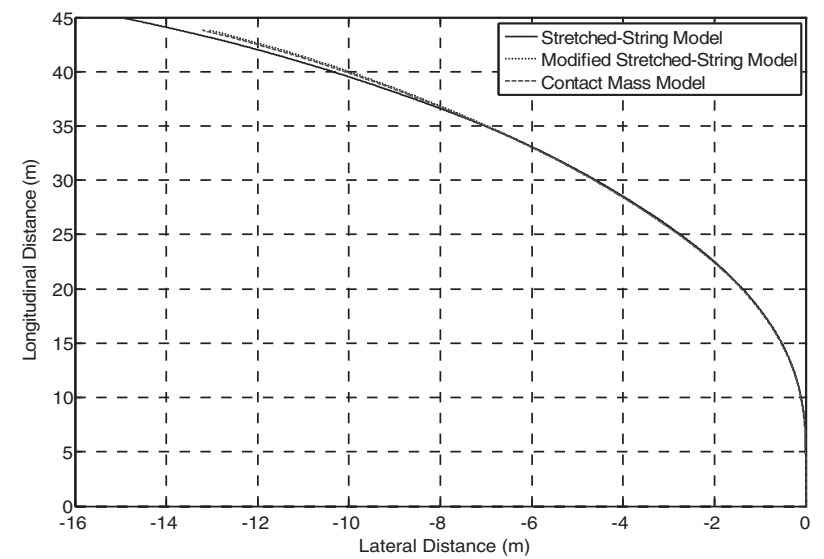

Fig. 17 Vehicle path curve for ABS braking while corner$\operatorname{ing}(V=70 \mathrm{~km} / \mathrm{h}$, dry)

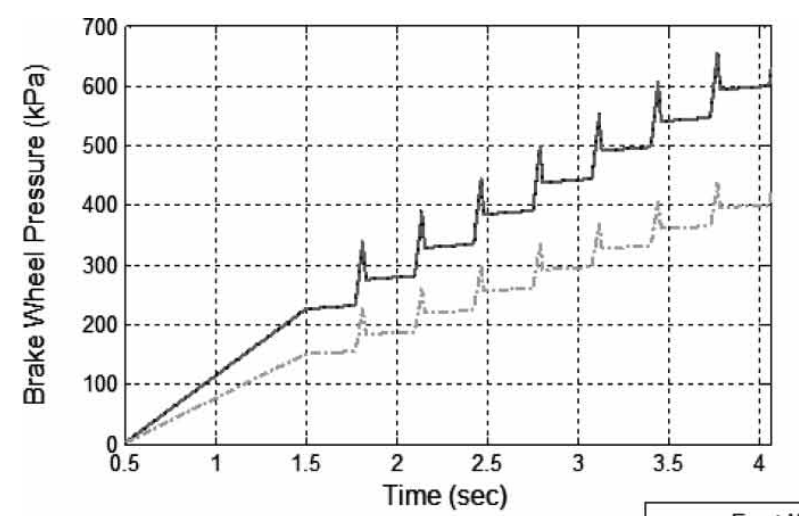

(a)

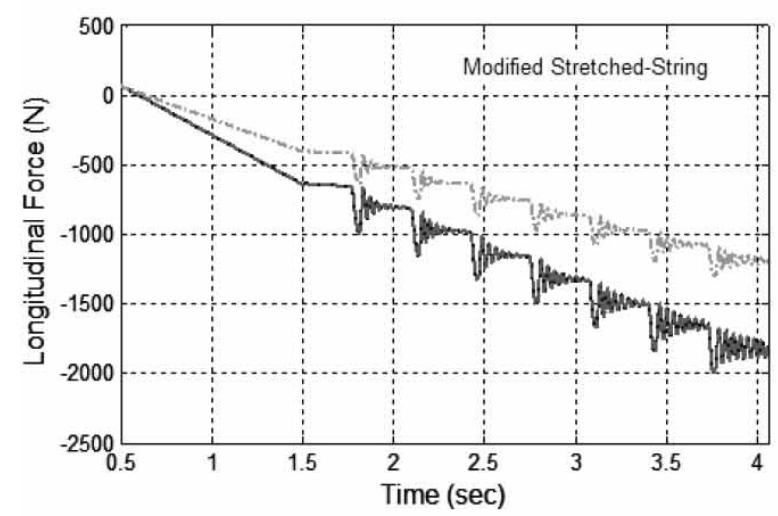

(c)
This promotes oscillations, as the higher value of relaxation length delays the force response. In the modified stretched-string tyre model, the relaxation length reduces and together with it the tendency of the tyre to oscillate.

As opposed to the ABS simulation performed earlier, where the modified stretched-string tyre model and the contact mass model showed similar behaviours,

Table 3 Average braking force comparison $(V=70 \mathrm{~km} / \mathrm{h}$, dry, braking with cornering)

\begin{tabular}{|c|c|c|c|c|}
\hline \multirow[b]{2}{*}{ Tyre models } & \multicolumn{4}{|c|}{$\begin{array}{l}\text { Average brake force at } \\
\text { individual wheels }(\mathrm{N})\end{array}$} \\
\hline & FL & FR & RL & $\mathrm{RR}$ \\
\hline Stretched-string transient tyre model & 2910 & 3808 & 1808 & 1806 \\
\hline Modified stretched-string tyre model & 3255 & 4801 & 1693 & 1669 \\
\hline Contact mass transient tyre model & 3281 & 4762 & 1678 & 1656 \\
\hline
\end{tabular}

Table 4 Braking distance for the three transient tyre models while cornering $(V=70 \mathrm{~km} / \mathrm{h})$

\begin{tabular}{llll}
\hline & \multicolumn{3}{c}{ Braking distance $(\mathrm{m})$} \\
\cline { 2 - 4 } Tyre models & Dry & Wet & Snow \\
\hline Stretched-string transient tyre model & 29.44 & 49.12 & 96.65 \\
Modified stretched-string tyre model & 25.5 & 42.86 & 89.01 \\
Contact mass transient tyre model & 25.55 & 42.87 & 88.87 \\
\hline
\end{tabular}

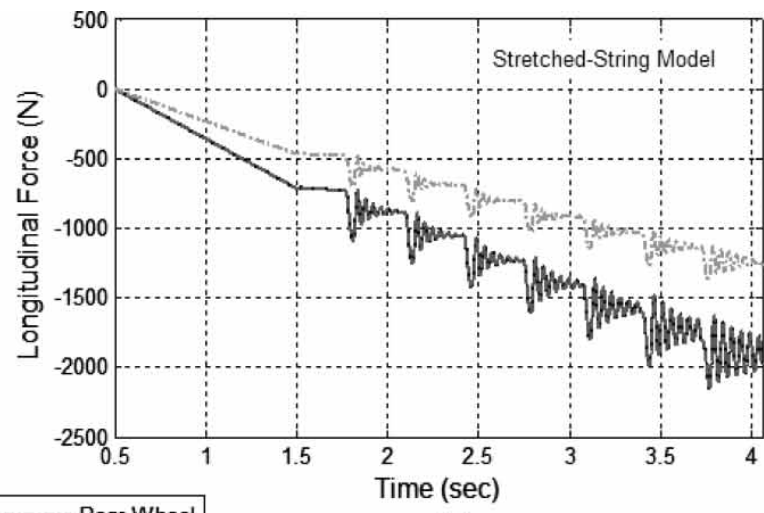

(b)

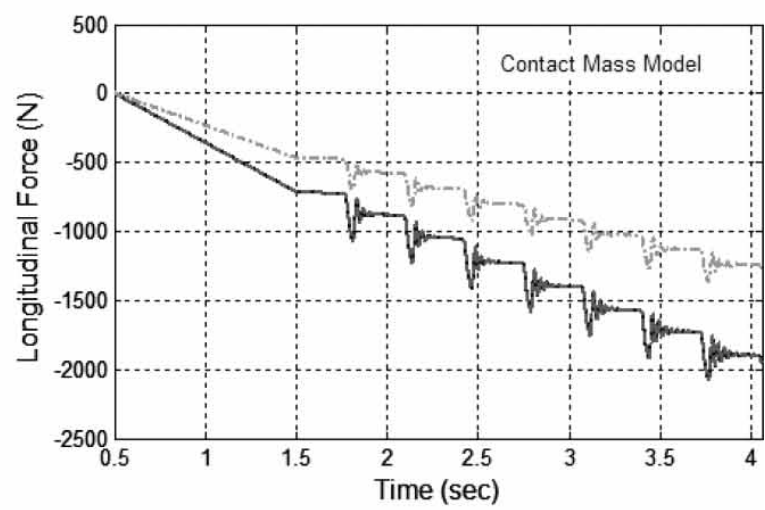

(d)

Fig. 18 Step braking response of the three transient tyre models $(V=34 \mathrm{~km} / \mathrm{h}$, dry, straight line) 
the longitudinal force response of the contact mass model in this case shows improved performance compared with the modified stretched-string tyre model (Fig. 18(d)). This can be attributed to the fact that the contact mass model includes additional damping in the carcass, which leads to further suppression of oscillations. As observed in these new set of simulations, except for the contact mass transient model, the other two tyre models exhibit large fluctuations, which take much longer time to settle. The highamplitude longitudinal force fluctuations may lead to wheel speed disturbances, eventually affecting the performance of the ABS system, as the controller may have to deal with inaccurate feedback signals.

\section{CONCLUSION}

The article has investigated the influence of tyre transient behaviour on the performance of an ABS system. By employing a representative ABS system and considering the particularly demanding combined braking and cornering manoeuvre, it is shown that the tyre models which are adequate for pure braking may not be sufficient when the complicated full vehicle dynamics are considered. In comparison with the earlier studies, which were mostly conducted for straightline braking, the article emphasizes required tyre model characteristics which can influence the complex interactions encountered, whose effects should be evaluated.

Transient dynamic behaviour of three tyre models are analysed in detail. The stretched-string tyre model is found not to accurately describe realistic damping arising in the contact patch. As a consequence, the model promotes higher tyre oscillations than would be expected. Unlike the stretched-string tyre model, the relaxation length in the modified stretched-string tyre model varies with tyre slip, such that with an increase in slip the relaxation length decreases. This means that the tyre response lag in both longitudinal as well as lateral directions reduces at higher slips, which eventually leads to better performance during ABS braking simulation. The contact mass model, in comparison, follows a slightly different approach, where instead of the normal relaxation length approach, the inclusion of single point contact mass along with the carcass springs represent the dynamic characteristics of a tyre in a more realistic manner. In the initial analysis, both the modified stretched-string tyre and the contact mass models produced similar performance during ABS simulation. However, in the second set of simulations, when a stepped brake pressure with short pulses were used as an input, the contact mass model exhibited reduced oscillatory behaviour and quicker response time to the brake pressure variations.

(C) Authors 2010

\section{REFERENCES}

1 Owen, R. H. and Bernard, J. E. Directional dynamics of a tractor-loader-backhoe. Veh. Syst. Dyn., 1982, 11, 251-265.

2 Ellis, J. R. Vehicle handling dynamics, 1994 (Mechanical Engineering Publications, London).

3 Loeb, J. S., Guenther, D. A., Chen, H.-H. F., and Ellis, J. R. Lateral stiffness, cornering stiffness and relaxation length of the pneumatic tire. In Proceedings of the SAE International Congress and Exposition, Detroit, Michigan, USA, 26 February-2 March 1990.

4 Heydinger, G. J., Garrott, W. R., and Chrstos, J. P. The importance of tire lag on simulated transient vehicle response. SAE technical paper 910235, 1991.

5 Bernard, J. E. and Clover, C. L. Tire modeling for lowspeed and high-speed calculations. SAE technical paper 950311, 1995.

6 Kuiper, E. and Van Oosten, J. J. M. The PAC2002 advanced handling tire model. Veh. Syst. Dyn., 2007, 45, 153-167.

7 Pacejka, H. B. Analysis of the dynamic response of a rolling string-type tire model to lateral wheel-plane vibrations. Veh. Syst. Dyn., 1972, 1, 37-66.

8 van der Jagt, P., Pacejka, H. B., and Savkoor, A. R. Influence of tyre and suspension dynamics on the braking performance of an anti-lock system on uneven roads. In Proceedings of the Second International Conference on New Developments in Powertrain and Chassis Engineering, Strasbourg, France, 14-16 June 1989.

9 Jansen, S. T. H., Zegelaar, P. W. A., and Pacejka, H. B. Influence of in-plane tyre dynamics on ABS braking of a quarter vehicle model. Veh. Syst. Dyn., 1999, 32, 249-261.

10 Pauwelussen, J. P., Gootjes, L., Schroder, C., Kohne, K.-U., Jansen, S., and Schmeitz, A. Full vehicle ABS braking using the swift rigid ring tyre model. Control Eng. Pract., 2003, 11, 199-207.

11 Braghin, F., Cheli, F., Giangiulio, E., Mancosu, F., and Arosio, D. A fast and reliable dynamic tyre-road contact model for abs braking manoeuvre simulations. In Proceedings of the Eighth Biennial ASME Conference on Engineering Systems Design and Analysis, ESDA2006, Torino, Italy, 4-7 July 2006.

12 van Zanten, A., Ruf, W. D., and Lutz, A. Measurement and simulation of transient tire forces. SAE technical paper 890640, 1989.

13 van Zanten, A., Erhardt, R., and Lutz, A. Measurement and simulation of transients in longitudinal and lateral tire forces. In Proceedings of the 1990 SAE International Congress and Exposition, Detroit, Michigan, USA, 26 February-2 March 1990.

14 Jaiswal, M., Mavros, G., Rahnejat, H., and King, P. D. A multi-body dynamics approach for the study of critical handling manoeuvres on surfaces with uneven friction. In Proceedings of the ECCOMAS Thematic Conference on Multi-body Dynamics, Milano, Italy, 25-28 June 2007.

15 Pacejka, H. B. Tyre and vehicle dynamics, 2006 (Butterworth-Heinemann, Oxford).

16 Azman, M., King, P. D., and Rahnejat, H. Combined bounce, pitch, and roll dynamics of vehicles negotiating single speed bump events. Proc. IMechE, Part K: J. Multi-body Dynamics, 2007, 221(K1), 33-40. DOI: 10.1243/1464419JMBD55. 
17 Happian-Smith, J. An introduction to modern vehicle design, 2002 (Butterworth-Heinemann, Oxford).

18 Bauer, H. Driving-safety systems, 1999 (Robert Bosch $\mathrm{GmbH}$, Stuttgart).

19 ADAMS/Tire user's manual, 2005 (Mechanical Dynamics Inc., Michigan, USA).

20 Pacejka, H. B. and Besselink, I. J. M. Magic formula tyre model with transient properties. In Proceedings of the 1997 Second International Colloquium on Tyre Models for Vehicle Dynamic Analysis, Berlin, Germany, 20-21 February 1997

21 Harned, J. L., Johnston, L. E., and Scharpf, G. Measurement of tire brake force characteristics as related to wheel slip (antilock) control system design. SAE technical paper 690214, 1969.

\section{APPENDIX}

\section{Notation}

$a, b$

$+A,+a,-a$

$c_{\mathrm{c} x}, c_{\mathrm{c} y}$

$C_{F x}, C_{F y}$

$C_{F \alpha}, C_{F \kappa}$

$\mathrm{d} f_{z}$

$D$

$F_{x}, F_{y}$

$F_{x a}, F_{y a}$

$F_{z}$

$F_{z 0}$

$h_{0}$

$I_{x x}, I_{y y}, I_{z z}$

$I_{x y}, I_{y z}, I_{z x}$

$k_{\mathrm{c} x}, k_{\mathrm{c} y}$

$m_{\mathrm{c}}$

$m_{\mathrm{s}}, m_{\mathrm{T}}$

$p, q, r$

$p_{\mathrm{Ky3}}$

$p_{\mathrm{T} x 1}$

$p_{\mathrm{T} x 2}$

$p_{\mathrm{T} x 3}$

$p_{\mathrm{T} y 1}$ distance of the front and rear axle

from the origin of the SAE frame of reference

threshold terms for wheel peripheral acceleration

longitudinal and lateral damping

longitudinal and lateral tyre stiffness

cornering and longitudinal slip

stiffness

non-dimensional vertical load

increment

peak factor of the magic formula tyre model

longitudinal and lateral tyre forces

longitudinal and lateral forces acting

on the wheel rim

tyre vertical load

reference nominal load

height of the SAE frame of reference

from the global fixed frame of

reference

mass moments of inertia

product moments of inertia

longitudinal and lateral stiffness

contact patch mass

sprung and total masses of the

vehicle

roll, pitch, and yaw rates of the

vehicle

variation of cornering

stiffness/nominal load with

inclination

longitudinal relaxation length at

nominal load

variation of longitudinal relaxation

length with load

variation of longitudinal relaxation

length with exponent of load

peak value of relaxation length for

lateral direction

\section{$p_{\mathrm{T} y 2}$}

$r_{0}, r_{e}$

$\boldsymbol{r}_{1-4}$

$t_{\mathrm{rf}}, t_{\mathrm{rr}}$

$u, v$

$u_{1}, v_{1}$

$U, V, W$

$U_{\mathrm{w}}, V_{\mathrm{w}}$

$V_{\mathrm{r}}$

$V_{\text {Ref }}$

$V_{\mathrm{s} x}, V_{\mathrm{s} y}$

$V_{\mathrm{s} x}^{*}, V_{\mathrm{s} y}^{*}$

$x_{\mathrm{G}}, y_{\mathrm{G}}, z_{\mathrm{G}}$

$X, Y, Z$

$z_{\text {body }_{1-4}}$

$z_{\text {wheel }_{1-4}}$

$\alpha, \kappa$

$\alpha^{\prime}, \kappa^{\prime}$

$\alpha_{\mathrm{f}}^{\prime}$

$\gamma$

$\gamma_{y}$

$\varepsilon_{\mathrm{F}}$

$\lambda_{1}$

$\sigma_{\mathrm{c}}$

$\sigma_{\kappa}, \sigma_{\alpha}$

$\sigma_{\kappa 0}, \sigma_{\alpha \mathrm{o}}$

$\sigma_{\kappa}^{*}, \sigma_{\alpha}^{*}$

$\Sigma F_{x, y, z}$

$\Sigma M_{x, y, z}$

$\varphi, \theta, \psi$

$\Omega$ shape factor for lateral relaxation length

free rolling and effective rolling radii of the tyre

position vector of the tyre contact patches from the SAE frame of reference

half track widths at the front and rear

longitudinal and lateral deflections of the carcass

longitudinal and lateral

displacement of the leading edge of the contact point

vehicle translational velocity relative to the SAE (moving) frame of reference

vehicle longitudinal and lateral velocities projected on the wheel plane

wheel peripheral velocity along the wheel plane

reference velocity

longitudinal and lateral slip velocities longitudinal and lateral slip velocities of the contact patch mass point coordinates of the position of the centre of mass of the vehicle

SAE frame of reference vertical displacements of the vehicle sprung body (four corners) vertical displacements of the wheels

steady-state slip terms transient slip terms effective slip angle which account for the shift $\Delta \alpha$

camber angle

camber variation

factor to avoid singularity while calculating transient slip

slip threshold

contact relaxation length

longitudinal and lateral relaxation lengths

initial longitudinal and lateral relaxation lengths longitudinal and lateral relaxation lengths for the modified stretched-string tyre model sum of forces in the direction of $x, y, z$ of the SAE frame of reference sum of moments in the direction of $x, y, z$ of the SAE frame of reference Euler angles rotational wheel speed 